\title{
Bulk flows in Virasoro minimal models with boundaries
}

\author{
Stefan Fredenhagen ${ }^{1}$, Matthias R Gaberdiel ${ }^{2}$ and \\ Cornelius Schmidt-Colinet ${ }^{2}$ \\ ${ }^{1}$ Max-Planck-Institut für Gravitationsphysik, Albert-Einstein-Institut, D-14424 Golm, Germany \\ ${ }^{2}$ Institut für Theoretische Physik, ETH Zürich, CH-8093 Zürich, Switzerland \\ E-mail: stefan@aei.mpg.de, gaberdiel@itp.phys.ethz.ch and schmidtc@itp.phys.ethz.ch
}

Received 7 September 2009, in final form 26 October 2009

Published 25 November 2009

Online at stacks.iop.org/JPhysA/42/495403

\begin{abstract}
The behaviour of boundary conditions under relevant bulk perturbations is studied for the Virasoro minimal models. In particular, we consider the bulk deformation by the least relevant bulk field which interpolates between the $m$ th and $(m-1)$ th unitary minimal model. In the presence of a boundary, this bulk flow induces an RG flow on the boundary, which ensures that the resulting boundary condition is conformal in the $(m-1)$ th model. By combining perturbative RG techniques with insights from defects and results about nonperturbative boundary flows, we determine the endpoint of the flow, i.e. the boundary condition to which an arbitrary boundary condition of the $m$ th theory flows to.
\end{abstract}

PACS numbers: $11.10 . \mathrm{Hi}, 11.10 . \mathrm{Kk}, 11.25 . \mathrm{Hf}$

(Some figures in this article are in colour only in the electronic version)

\section{Introduction}

Perturbations of conformal field theories by marginal or relevant operators play an important role in various contexts, for example in string theory where they describe string moduli or time-dependent processes. Many aspects of perturbed conformal field theories have been studied over the years, starting from the seminal work of Zamolodchikov [1, 2], and a number of structural results are known, in particular the $c$-theorem for bulk perturbations [3], as well as the $g$-theorem for boundary perturbations $[4,5]$.

Most of the work so far has been done on bulk perturbations of bulk conformal field theories, or boundary perturbations of boundary conformal field theories. However, it is clear that a bulk perturbation will also affect the boundary condition since the boundary condition of the original theory will typically not be conformal with respect to the new bulk conformal fixed point. This combined problem has only recently been addressed from the point of view 
of perturbed conformal field theory [6-8] (see also [9, 10]), although there has been earlier work in the context of integrable models starting from [11] and further developed in [12-14]. In particular, these flows have been studied using a version of the thermodynamic Bethe ansatz (see for example [15-19]), the truncated conformal space approach (see for example [17, 18, 20]) and a form factor expansion [21,22]. From the point of view of perturbed conformal field theory, a bulk perturbation generically induces a boundary renormalization group (RG) flow that will ensure that at the endpoint of the flow, both bulk and boundary are again conformal [6-8].

The resulting coupled RG equations have so far only been worked out for a few simple examples. In all of them, the bulk perturbation was actually exactly marginal in the bulk. As a consequence, the bulk RG equation was trivial, and one only had to solve the boundary RG equation with a (bulk) source term. In this paper, we shall demonstrate that these techniques also work nicely for a genuinely coupled bulk boundary problem, where neither of the perturbations is marginal.

The archetypal examples for which these kinds of problems can be studied are the Virasoro minimal models. Indeed, the original analysis of Zamolodchikov [1] was performed in this context: he established that the perturbation of the $m$ th minimal model by the least relevant field, the bulk field $\phi_{(1,3)}$, induces an RG flow whose endpoint is the $(m-1)$ th minimal model. The analogous analysis for the boundary perturbation - the perturbation of a Cardy boundary condition [23] of the $m$ th minimal model by the $\psi_{(1,3)}$ boundary field-was done in [24]. They showed that the endpoint of this boundary flow is in general a superposition of fundamental boundary conditions. For the combined problem, the bulk perturbation by $\phi_{(1,3)}$ in the presence of a boundary, only a few numerical studies have been performed so far $[25,26]$, and some conjectural TBA results exist $[16,27]$. In this paper, we will fill in this gap and show how this problem can be analysed analytically.

Let us briefly sketch our argument. The bulk RG equation is unaffected by the presence of the boundary, and thus the old fixed point analysis of Zamolodchikov applies. The boundary $\mathrm{RG}$ equation, on the other hand, is of the form

$$
\dot{\mu}=(1-h) \mu+\frac{1}{2} B \lambda+D \mu^{2}+E \lambda \mu+F \lambda^{2}+\mathcal{O}\left(\mu^{3}, \lambda \mu^{2}, \lambda^{2} \mu, \lambda^{3}\right) .
$$

Here the first and the third terms are the usual boundary RG equation terms for the boundary coupling $\mu$, while the other three terms also involve the bulk coupling constant $\lambda$. The $B \lambda$ term is the source term that was studied in [6], whereas the $E \lambda \mu$ term describes how the bulk deformation modifies the conformal weight of the boundary field [8]. In some sense, this term only appears at higher order in perturbation theory, and it was only recently understood how to calculate it as an integral of a chiral four-point function [8]. The $F \lambda^{2}$ term has not so far been studied in detail, but the coefficient $F$ itself is parametrically small-it is of order $1 / m$-and thus the $F \lambda^{2}$ term is subleading. In fact, our analysis will be performed for large $m$, for which we shall find perturbative fixed points for $\lambda$ and $\mu$ that are of order $1 / m$. The $E \lambda \mu$ term is then of the same order as the standard $D \mu^{2}$ term, while the $F \lambda^{2}$ will be subleading and can be ignored to leading order.

The resulting RG equations have generically three perturbative fixed points: the pure boundary perturbation fixed point of [24], as well as two perturbative fixed points in the $(m-1)$ th theory (i.e. for non-trivial $\lambda)$. The first fixed point (I) can be identified as in [24], namely by computing the perturbed $g$-function and identifying it with the $g$-function of the fixed point boundary condition. However, the analysis for the other two fixed points (II and III) is not so straightforward since they live in a different bulk theory than the one we started with, and it is therefore not clear to what extent we can compare the $g$-functions directly. However, it is reasonable to assume that it makes sense to compare ratios of $g$-functions [18], and it is 


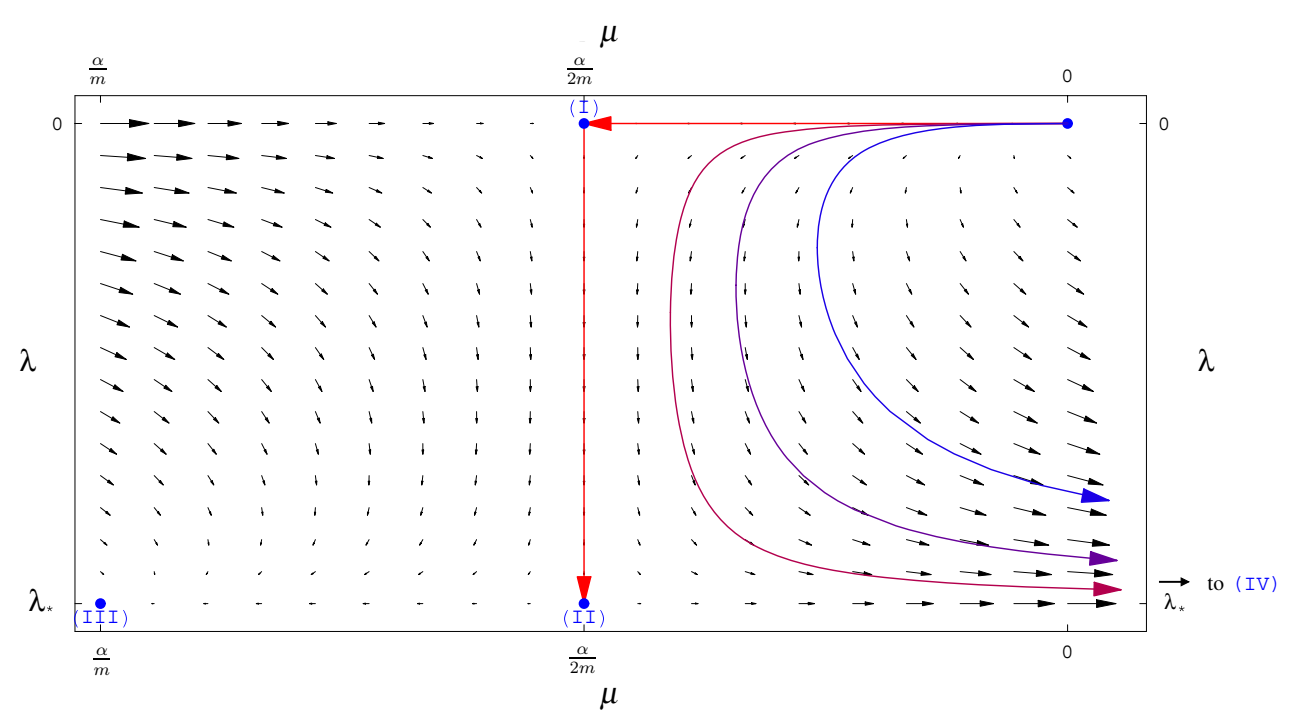

Figure 1. The combined flow diagram for $\left(a_{1}, a_{2}\right)=(2,3)$ and $m=100$ (for which $\alpha=-4$; see section 2.2 for details). We have magnified the vectors $(\dot{\mu}, \dot{\lambda})$ by a factor 2.5 . The horizontal arrow indicates the pure boundary flow to the perturbative fixed point (I) in the $m$ th minimal model, the vertical arrow describes the flow of the boundary condition (I) to the boundary condition (II) in the $(m-1)$ th minimal model. The three other flows that are depicted are generic bulk-boundary RG-flows that at the end tend towards the fixed point (IV) where $\mu=+\infty$ in the $(m-1)$ th minimal $\operatorname{model}\left(\lambda=\lambda_{*}\right)$.

furthermore plausible that the boundary condition with the overall smallest $g$-function-this is the boundary condition that corresponds to the identity representation-should flow to the corresponding boundary condition in the $(m-1)$ th theory. This tells us how the overall scale of the $g$-functions changes, and thus allows us to make a definite prediction for the $g$-function of the fixed point in the $(m-1)$ th minimal model. Progressing in this manner, we can then identify the two perturbative fixed points in the $(m-1)$ th theory. As it turns out, one of the fixed points (III) is actually the endpoint of a pure $\psi_{(1,3)}$ boundary perturbation of the other (II) [28, 29], in agreement with the general structure of our RG flow diagram (see figure 1).

As is clear from this diagram, neither of these perturbative fixed points can be reached by a generic RG flow: we can only get to the unstable fixed point (II) if we first perform the pure boundary flow to (I), followed by a pure bulk flow, and we can only get to (III) via (II). However, we can read off the actual endpoint of a generic flow from this picture: it is the endpoint of the pure boundary flow starting from (II), but flowing in the opposite direction to (III). The resulting fixed point is therefore the non-perturbative fixed point of a certain boundary perturbation of (II). At least in some specific cases, this fixed point has been identified before using TCSA and TBA techniques [16, 27, 30]; applying these results we can therefore make a prediction for the actual fixed point (IV) of our RG flow at least for some restricted set of initial boundary conditions.

In order to determine the corresponding fixed point for an arbitrary initial boundary condition, we finally use techniques from the perturbation theory of topological defects. In particular, we can identify the RG fixed point of a certain topological defect from the above boundary flow results (that we know for a restricted set of initial conditions). We can then use the RG flow behaviour of this topological defect in order to make a prediction for the 
ultimate (IV) fixed point of an arbitrary initial boundary condition. The resulting predictionsee equation (4.20) - is the main result of our paper. As we shall demonstrate, it satisfies a number of consistency conditions; in particular, the flows for different initial boundary conditions actually organize themselves into long chains (see figure 2 in section 4.3), some of whose individual flows were known before. In addition, we are able to confirm this prediction, for a particular class of boundary conditions (namely those near the middle of the Kac table), by a direct perturbative calculation-see section 4.4. As a final check of our prediction, we extrapolate it to small values of $m$ and show that our results are consistent with the numerical results of $[25,26]$.

The paper is organized as follows. The coupled RG equations are worked out in section 2.1. In section 2.2 we identify the various perturbative fixed points and study the structure of the RG flow diagram. To support the identification of the fixed points, we then perform a detailed analysis of the perturbed $g$-function in section 3 . In section 4 , we combine these results with insights from non-perturbative flows and constraints coming from the action of defects to give a complete picture of the flow diagram, including also the actual (non-perturbative) fixed point of the original flow (for a generic initial condition). We also discuss there various consistency checks which our analysis satisfies. Finally, we compare our findings with the numerical study of $[25,26]$ in section 5 . There are three appendices, where we collect the leading behaviour of the various OPE coefficients (appendix A), give explicit formulae for the bulk and boundary correlation functions on the upper half-plane and the disc (appendix B), and analyse the explicit solutions to the RG equations (appendix C).

\section{RG equations for minimal models}

Let us begin by reviewing our conventions. We shall consider the unitary minimal models with central charge

$$
c_{m}=1-\frac{6}{m(m+1)}, \quad m=3,4,5, \ldots
$$

More specifically, we shall always work with the diagonal (charge conjugation) modular invariant theory, for which the left- and the right-moving representations are identical. For $c=c_{m}$, the allowed irreducible highest weight representations of the Virasoro algebra have highest weight:

$$
h_{(r, s)}=\frac{((m+1) r-m s)^{2}-1}{4 m(m+1)},
$$

where $1 \leqslant r \leqslant m-1$ and $1 \leqslant s \leqslant m$, and we have the identifications $(r, s) \cong(m-r, m+1-s)$. For the charge conjugation theory, the conformal primary fields $\phi_{i}$ are labelled by $i \equiv(r, s)$, and the conformal dimension of $\phi_{i}$ is $\Delta_{i}=2 h_{i}$.

We shall be interested in the conformal field theory defined on a Riemann surface with boundary, more specifically the upper half-plane (or equivalently the disc). In order to characterize the theory on a surface with boundary, we also have to specify the boundary conditions for the various fields. For the diagonal modular invariant theories, the possible conformal boundary conditions are also parameterized by the highest weight representations of the Virasoro algebra. Thus, the most general conformal boundary condition is a superposition of boundary conditions associated with the irreducible representations labelled by $\left(a_{1}, a_{2}\right)$, where $a_{1}$ and $a_{2}$ have the same ranges and identifications as $r$ and $s$ in (2.2). It is sometimes 
convenient to describe the boundary condition in terms of the associated boundary state; for the boundary condition $\mathbf{a}=\left(a_{1}, a_{2}\right)$, the boundary state is given by the Cardy formula [23]

$$
\left.\left.\left.\| \mathbf{a}\rangle\rangle \equiv \| a_{1}, a_{2}\right\rangle\right\rangle=\sum_{(r, s)} \frac{S_{\left(a_{1}, a_{2}\right)}^{(r, s)}}{\sqrt{S_{(1,1)}^{(r, s)}}}|r, s\rangle\right\rangle .
$$

Here $|r, s\rangle\rangle$ denotes the Ishibashi state [31] in the $(r, s)$ sector, and $S_{\left(a_{1}, a_{2}\right)}{ }^{(r, s)}$ is the modular $S$-matrix, whose entries are explicitly given as

$S_{\left(a_{1}, a_{2}\right)}^{(r, s)}=\sqrt{\frac{8}{m(m+1)}}(-1)^{1+a_{1} s+a_{2} r} \sin \left(\frac{m+1}{m} \pi a_{1} r\right) \sin \left(\frac{m}{m+1} \pi a_{2} s\right)$.

In the presence of a boundary, there are also excitations located at the boundary. These are described by boundary fields $\psi_{i}$, and they are again characterized by unitary representations of the Virasoro algebra, $i \equiv(r, s)$. The conformal dimension of the boundary field $\psi_{i}$ is given by the weight $h_{i}$ as in (2.2). On the boundary condition a, the possible boundary fields are those that appear in the fusion rules of $\mathbf{a}$ with itself.

In the following section, we shall work on the upper half-plane, where we denote the operator product expansions (OPEs) of the bulk and boundary fields as

$$
\begin{aligned}
& \phi_{i}\left(z_{1}, \bar{z}_{1}\right) \phi_{j}\left(z_{2}, \bar{z}_{2}\right)=\sum_{k} C_{i j}{ }^{k} \phi_{k}\left(z_{2}, \bar{z}_{2}\right)\left|z_{1}-z_{2}\right|^{\Delta_{k}-\Delta_{i}-\Delta_{j}}+\cdots, \\
& \phi_{i}(z=x+\mathrm{i} y, \bar{z})=\sum_{k} B_{i}{ }^{k} \psi_{k}(x)(2 y)^{h_{k}-\Delta_{i}}+\cdots, \\
& \psi_{i}(x) \psi_{j}(y)=\sum_{k} D_{i j}{ }^{k} \psi_{k}(y)(x-y)^{h_{k}-h_{i}-h_{j}}+\cdots \quad(x>y) .
\end{aligned}
$$

\subsection{Bulk perturbation}

We are interested in the perturbation of the conformal field theory by the least relevant bulk field $\phi_{(1,3)}$ of conformal weight $h \equiv h_{(1,3)}=\frac{m-1}{m+1}$. As is well known, this perturbation induces an RG flow that drives the minimal model $c_{m}$ to the one corresponding to $c_{m-1}[1,32]$. In this paper, we study what happens if we consider this perturbation in the presence of a boundary. It is clear that the presence of the boundary will not affect the flow in the bulk, i.e. that we are still ending up with the minimal model corresponding to $c_{m-1}$. However, it is not so clear what happens to the boundary condition $\left(a_{1}, a_{2}\right)$ of the $c_{m}$ theory under the RG flow; this is the question we want to address in the following.

In the presence of a boundary, the RG flow will also switch on boundary fields, and we should therefore consider the general perturbation

$$
\delta S=\sum_{k} \lambda_{k} \epsilon^{\Delta_{k}-2} \int \mathrm{d}^{2} z \phi_{k}(z, \bar{z})+\sum_{l} \mu_{l} \epsilon^{h_{l}-1} \int \mathrm{d} x \psi_{l}(x) .
$$

Here the $\lambda_{k}$ and the $\mu_{l}$ are (small) dimensionless coupling constants, and $\epsilon$ is an ultraviolet cut-off. As has been studied before, the combined RG equations are [6, 8]

$$
\begin{gathered}
\dot{\lambda}_{k}=\left(2-\Delta_{k}\right) \lambda_{k}+\sum_{i j} \pi C_{i j}{ }^{k} \lambda_{i} \lambda_{j}+\mathcal{O}\left(\lambda^{3}\right), \\
\dot{\mu}_{l}=\left(1-h_{l}\right) \mu_{l}+\sum_{i} \frac{1}{2} B_{i}{ }^{l} \lambda_{i}+\sum_{i j} E_{i j}{ }^{l} \lambda_{i} \mu_{j}+\sum_{i j} D_{i j}{ }^{l} \mu_{i} \mu_{j} \\
+\sum_{i j} F_{i j}{ }^{l} \lambda^{i} \lambda^{j}+\mathcal{O}\left(\mu^{3}, \lambda^{2} \mu, \lambda \mu^{2}\right),
\end{gathered}
$$


where $\dot{\lambda}$ stands for $\mathrm{d} \lambda / \mathrm{d} \log \epsilon$, and similarly for $\dot{\mu}$. In the OPE scheme that we shall consider in the following ${ }^{3}$, the coefficients $C_{i j}{ }^{k}, B_{i}{ }^{l}$ and $D_{i j}{ }^{l}$ are those from the OPEs (2.5). Furthermore, the coefficient $E_{i j}^{l}$ can be calculated in terms of an integral of a four-point function [8] (see below for more details), while the coefficient $F_{i j}^{l}$ comes from the correlation function of two bulk fields and one boundary field. As will be explained below, this contribution is only subleading.

Note that we have written out explicitly all terms that are of degree less than or equal to 2 in $\lambda$ and $\mu$. We are interested in a perturbative fixed point for $\lambda$ and $\mu$, for which both are of order $1 / \mathrm{m}$. The terms we have spelled out are therefore all the terms that contribute up to order $1 / \mathrm{m}^{2}$. In particular, it is important that we also include the $\lambda \mu$ term for the $\mu \mathrm{RG}$ equation since it is of the same order as the $\mu^{2}$ term. (In fact, if one leaves it out, one does not find any perturbative fixed point for $\mu$.)

The perturbation by the $(1,3)$ bulk field is particularly tractable since the successive OPEs of $\phi_{(1,3)}$ with itself only contain one relevant field apart from the identity, namely $\phi_{(1,3)}$ itself. As a consequence, we can restrict the $\mathrm{RG}$ equation to $\lambda \equiv \lambda_{(1,3)}$, and the bulk equation is therefore

$$
\dot{\lambda}=(2-2 h) \lambda+\pi C_{(1,3)(1,3)}(1,3) \lambda^{2}+\cdots
$$

This leads to the well-known fixed point $[1,32] \lambda_{*}=-\frac{1}{\pi m}+\mathcal{O}\left(m^{-2}\right)$, where we have used the leading behaviour of the OPE coefficients as given in appendix A.

In the presence of a boundary $\mathbf{a}=\left(a_{1}, a_{2}\right)$, the bulk perturbation also induces boundary perturbations, and the only relevant boundary field (apart from the identity) that is switched on is the boundary $\psi_{(1,3)}$ field. Furthermore, successive OPEs of $\psi_{(1,3)}$ generate, apart from the identity field, only one relevant field, namely $\psi_{(1,3)}$ itself. It is therefore again consistent to restrict our attention to this field ${ }^{4}$. Writing $\mu=\mu_{(1,3)}$, the $\mathrm{RG}$ equations are

$\dot{\mu}=(1-h) \mu+\frac{1}{2} B_{(1,3)}^{(1,3)} \lambda+E_{(1,3)(1,3)}^{(1,3)} \lambda \mu+D_{(1,3)(1,3)}^{(1,3)} \mu^{2}+F \lambda^{2}+$ higher order.

The leading behaviour of the coefficients $B$ and $D[33]$ is given in appendix A.1, while $E$ can be calculated following [8] as

$$
\begin{aligned}
E_{(1,3)(1,3)}^{(1,3)}=\lim _{\epsilon \rightarrow 0} & \frac{1}{2} \int \mathrm{d} x \theta\left(\frac{1}{\epsilon}-|x|\right)\left[\frac{1}{\langle\mathbb{1}\rangle D_{(1,3)(1,3)}^{(1,1)}}\right. \\
& \times\left\langle\phi_{(1,3)}\left(x+\frac{\mathrm{i}}{2}, x-\frac{\mathrm{i}}{2}\right) \psi_{(1,3)}(0) \psi_{(1,3)}(\infty)\right\rangle \\
& \left.-B_{(1,3)}^{(1,1)} D_{(1,1)(1,3)}^{(1,3)}-B_{(1,3)}^{(1,3)} D_{(1,3)(1,3)} \frac{\theta(|x|-1)}{|x|^{h}}\right] .
\end{aligned}
$$

The correlator in the first line of this expression is a chiral four-point function which is in principle determined by the differential equation that comes from the null vector descendant of the highest weight state with $h=h_{(1,3)}$. Unfortunately, this differential equation does not seem to have a simple solution. However, we are only interested in the chiral four-point function to leading order in $1 / \mathrm{m}$, and in this limit we find

$$
\frac{1}{\langle\mathbb{1}\rangle D_{(1,3)(1,3)}^{(1,1)}}\left\langle\phi_{(1,3)}(z, \bar{z}) \psi_{(1,3)}(0) \psi_{(1,3)}(\infty)\right\rangle=\frac{\frac{1}{2}(z-\bar{z})^{4}-\frac{3}{2}\left(z^{4}+\bar{z}^{4}\right)}{|z|^{4}(z-\bar{z})^{2}}+\mathcal{O}\left(m^{-1}\right) \text {. }
$$

3 To leading order in $1 / m$, the calculation is actually the same as in a minimal subtraction scheme that is more convenient for the calculation of the perturbed $g$-function.

4 Obviously, this only makes sense if $\psi_{(1,3)}$ appears in the boundary spectrum of the boundary condition $\mathbf{a}=\left(a_{1}, a_{2}\right)$; this is the case provided that $1<a_{2}<m$. 
Some remarks on the calculation can be found in appendix B. Note that this function has the correct asymptotic behaviour as $z$ approaches the boundary away from the origin, because in this limit it goes as $\sim \frac{3}{4 y^{2}}$, which is the expected behaviour since (see appendix A.1)

$$
B_{(1,3)}^{(1,1)} D_{(1,1)(1,3)}^{(1,3)}=3+\mathcal{O}\left(m^{-1}\right)
$$

The first term in the second line in (2.10) subtracts precisely this leading term. On the other hand, the other channels do not contribute to leading order in $1 / \mathrm{m}$ since (see again appendix A.1)

$$
B_{(1,3)}^{(1,3)} D_{(1,3)(1,3)}^{(1,3)}=\mathcal{O}\left(m^{-1}\right), \quad B_{(1,3)}^{(1,5)} D_{(1,5)(1,3)}^{(1,3)}=\mathcal{O}\left(m^{-2}\right) .
$$

In particular, the integral thus converges, and we find explicitly the rather simple result

$$
E_{(1,3)(1,3)}^{(1,3)}=\frac{1}{2} \int_{-\infty}^{\infty} \mathrm{d} x\left[\frac{48 x^{4}-72 x^{2}-5}{\left(1+4 x^{2}\right)^{2}}-3\right]+\mathcal{O}\left(m^{-1}\right)=-4 \pi+\mathcal{O}\left(m^{-1}\right) .
$$

Note that the leading order result (in $1 / m$ ) is finite, and apparently scheme independent. This ties in with the general observation of [8] that $E$ is universal provided that the resonance condition is satisfied, which is here the case to leading order in $1 / \mathrm{m}$.

Finally, the term proportional to $F$ is subleading relative to these terms since it arises from the correlation function of two bulk fields and one boundary field. This correlation function is of the asymptotic form

$$
\begin{aligned}
\frac{1}{\langle\mathbb{1}\rangle D_{(1,3)(1,3)}^{(1,1)}}\langle & \left.\phi_{(1,3)}\left(z_{1}, \bar{z}_{1}\right) \phi_{(1,3)}\left(z_{2}, \bar{z}_{2}\right) \psi_{(1,3)}(\infty)\right\rangle \\
& \sim \sum_{\mathbf{i}, \mathbf{j}} B_{(1,3)} B_{(1,3)}^{\mathbf{i}} D_{\mathbf{i j}}{ }^{(1,3)} f_{\mathbf{i j}}\left(z_{1}, \bar{z}_{1}, z_{2}, \bar{z}_{2}\right),
\end{aligned}
$$

where $f_{\mathbf{i j}}\left(z_{1}, \bar{z}_{1}, z_{2}, \bar{z}_{2}\right)$ are some functions that give the asymptotic dependence on the insertion points, and $\mathbf{i}, \mathbf{j}=(1,1),(1,3),(1,5)$. With the OPE coefficients from appendix A.1, one can see that the contributions from all channels are at most of order $1 / \mathrm{m}$. The total contribution of the $F \lambda^{2}$ term is therefore subleading relative to the other terms.

\subsection{Analysis of fixed points}

Putting everything together, we thus have the coupled RG equations

$$
\begin{aligned}
& \dot{\lambda}=\frac{4}{m} \lambda+4 \pi \lambda^{2}+\mathcal{O}\left(\lambda^{3}\right) \\
& \dot{\mu}=\frac{2}{m} \mu+\frac{2 \pi \alpha}{m} \lambda-4 \pi \lambda \mu-\frac{4}{\alpha} \mu^{2}+\mathcal{O}\left(\lambda^{2} \mu, \lambda \mu^{2}, \mu^{3}\right),
\end{aligned}
$$

where

$$
\alpha= \begin{cases}\left(a_{1}-a_{2}\right)\left(a_{2}+1\right) & a_{2}>a_{1} \\ \frac{\left(a_{2}^{2}-1\right)}{m} & a_{2}=a_{1} \\ \left(a_{1}-a_{2}\right)\left(a_{2}-1\right) & a_{1}>a_{2}>1 .\end{cases}
$$

These equations hold provided that $a_{2}>1$. Otherwise, the boundary theory does not contain the relevant $\psi_{(1,3)}$ field, and there is therefore no equation for $\dot{\mu}$.

Apart from the trivial fixed point $(\lambda=\mu=0)$, these equations have the following three fixed points (for $a_{2}>1$ ). 
(I) The fixed point at

$$
\lambda_{*}=0, \quad \mu_{*}=\frac{\alpha}{2 m} .
$$

This is simply the perturbative fixed point in the pure boundary analysis of [24] (see also [28]). As was explained there, it describes the flow

$$
\left(a_{1}, a_{2}\right)_{m} \longrightarrow \bigoplus_{l=1}^{\min \left(a_{1}, a_{2}\right)}\left(a_{1}+a_{2}+1-2 l, 1\right)_{m}
$$

The endpoint of the flow is a boundary condition in the $m$ th theory.

(II) The fixed point at

$$
\lambda_{*}=-\frac{1}{\pi m}, \quad \mu_{*}=\frac{\alpha}{2 m} .
$$

The interpretation of this fixed point will be determined in detail in section 3 , where we will show that it describes the superposition of boundary conditions in the $(m-1)$ th theory

$$
\left(a_{1}, a_{2}\right)_{m} \longrightarrow \bigoplus_{l=1}^{\min \left(a_{1}, a_{2}\right)}\left(1, a_{1}+a_{2}+1-2 l\right)_{m-1} .
$$

(III) The fixed point at

$$
\lambda_{*}=-\frac{1}{\pi m}, \quad \mu_{*}=\frac{\alpha}{m} .
$$

As we will also show in section 3, this fixed point describes the endpoint of a perturbative boundary flow in the $(m-1)$ th theory, starting from the boundary condition at (II). The endpoint describes the boundary condition

$$
\left(a_{1}, a_{2}\right)_{m} \stackrel{\text { via (II) }}{\longrightarrow}\left(a_{2}, a_{1}\right)_{m-1},
$$

in agreement with a boundary flow of [28], where it was observed that by turning on all $\psi_{(1,3)}$ boundary condition changing fields, one can find the following two perturbative flows:

$$
\bigoplus_{l=1}^{\min \left(a_{1}, a_{2}\right)}\left(1, a_{1}+a_{2}+1-2 l\right)_{m-1} \longrightarrow\left\{\begin{array}{l}
\left(a_{2}, a_{1}\right)_{m-1} \\
\left(a_{1}, a_{2}\right)_{m-1}
\end{array}\right.
$$

(see equations (5.29) and (5.30) in [28]).

The above analysis applies to the case when both labels $a_{1}$ and $a_{2}$ are small, and $a_{2}>1$. If $a_{2}=1$, on the other hand, there is just the RG flow for $\lambda$, whose fixed point is $\lambda_{*}=-\frac{1}{\pi m}$. As will also be explained in section 3 , it describes the flow

$$
\left(a_{1}, 1\right)_{m} \longrightarrow\left(1, a_{1}\right)_{m-1} \text {. }
$$

It is easy to see from the flow diagram in the introduction (see figure 1) that for $a_{2}>1$, the actual flow cannot directly reach the fixed points (II) or (III). In fact, starting from $\lambda=\mu=0$, we do not get to the fixed point (II), unless we first perform the pure boundary flow leading to (I), followed by the pure bulk flow (2.25). To reach the fixed point (III), we first have to go to (II) via (I), and then have to switch on a pure boundary perturbation at (II). Indeed, the fixed point (II) is again unstable since the boundary condition (2.21) has at least one relevant $\psi_{(1,3)}$ field in its spectrum. This can also be seen by expanding the RG equation around the fixed point (II) by setting $\lambda=\lambda_{*}$ and $\mu=\alpha / 2 m+\tilde{\mu}$ :

$$
\left.\dot{\tilde{\mu}}\right|_{\lambda=\lambda_{*}}=\frac{2}{m} \tilde{\mu}-\frac{4}{\alpha} \tilde{\mu}^{2}+\cdots .
$$


The coefficient of the term linear in $\tilde{\mu}$ allows us to read off the conformal weight of the boundary field to which $\tilde{\mu}$ couples, and one finds indeed $h_{\tilde{\mu}}=h_{(1,3)}=1-\frac{2}{m}+\cdots$.

Given the various different kinds of flows we can consider, our resulting picture will have to satisfy a number of consistency conditions. These will be discussed in section 4, where we shall also analyse the actual non-perturbative fixed point (IV) to which a generic initial configuration will flow. Before we discuss these issues, let us first analyse the perturbed $g$-function in order to identify the different perturbative fixed points.

\section{Analysis of the perturbed $g$-function}

In order to corroborate our above claims about the perturbative fixed points, we shall now calculate the perturbed boundary entropy, as was done for the case of the pure boundary perturbation in [24]. In order to be able to compare with their results, we shall now work on the disc.

Recall that the boundary entropy $g(\mathbf{a})$ of a boundary condition $\mathbf{a}$ is defined to be the correctly normalized one-point function of the identity operator in the presence of the boundary condition a [4]:

$$
g^{(m)}(\mathbf{a})=\frac{S_{\mathbf{a}}^{\mathbb{1 1}}}{\sqrt{S_{1}^{\mathbb{1 1}}}}=\left(\frac{8}{m(m+1)}\right)^{\frac{1}{4}} \frac{\sin \frac{\pi a_{1}}{m} \sin \frac{\pi a_{2}}{m+1}}{\left(\sin \frac{\pi}{m} \sin \frac{\pi}{m+1}\right)^{\frac{1}{2}}} .
$$

It was conjectured in [4] and perturbatively verified in [24, 43] that this quantity decreases under pure boundary RG flows. Obviously, the same need not be true in our context, since our perturbation also changes the bulk theory, and we are therefore comparing one-point functions in different bulk models [18] (see also [44]).

We should thus not expect to be able to say much about the overall $g$-functions. On the other hand, the relative $g$-functions should continue to have a well-defined meaning (see also the discussion in [18]). Furthermore, we expect that the boundary condition $\mathbf{a}=(1,1)$ should flow to itself, since the boundary spectrum of the $(1,1)$ boundary does not contain any relevant fields (apart from the identity), and since $(1,1)$ is the boundary condition with the smallest $g$-function. Thus, it is natural to consider the relative boundary entropy with respect to $\mathbf{a}=(1,1)$ :

$$
\hat{g}^{(m)}(\mathbf{a})=\frac{g^{(m)}(\mathbf{a})}{g^{(m)}(1,1)}=\frac{\sin \frac{\pi a_{1}}{m} \sin \frac{\pi a_{2}}{m+1}}{\sin \frac{\pi}{m} \sin \frac{\pi}{m+1}} .
$$

For the following it will be important to consider the asymptotic expansion of the $g$-function for large $m$ with fixed boundary labels $a_{1}, a_{2}$ :

$\hat{g}^{(m)}(\mathbf{a})=a_{1} a_{2}\left(1-\frac{\pi^{2}}{6}\left(a_{1}^{2}+a_{2}^{2}-2\right) \frac{1}{m^{2}}+\frac{\pi^{2}}{3}\left(a_{2}^{2}-1\right) \frac{1}{m^{3}}+\mathcal{O}\left(m^{-4}\right)\right)$.

Our aim is therefore to calculate the perturbed $g$-function of the boundary condition a up to order $1 / \mathrm{m}^{3}$, and to deduce from it the perturbed value of the relative $g$-function (up to this order). This should then be identified with the relative $g$-function (in the $c_{m-1}$ theory) of the boundary condition to which a flows. Let us first deal with the case where $a_{2}>1$, so that the original boundary condition has a relevant boundary field in its spectrum. 


\subsection{The analysis for $a_{2}>1$}

Since we are interested in ratios of $g$-functions, it is convenient to consider the logarithm of the perturbed $g$-function

$$
\begin{aligned}
\log \left\langle\mathrm{e}^{\delta S}\right\rangle_{\mathbf{a}}=\log \langle\mathbb{1}\rangle_{\mathbf{a}} & +\frac{1}{\langle\mathbb{1}\rangle_{\mathbf{a}}}\left(\frac{1}{2} \mu_{*}^{2} \epsilon^{2 h-2} \int \mathrm{d} w_{1} \mathrm{~d} w_{2}\left\langle\psi\left(w_{1}\right) \psi\left(w_{2}\right)\right\rangle_{\mathbf{a}}^{c}\right. \\
& +\frac{1}{6} \mu_{*}^{3} \epsilon^{3 h-3} \int \mathrm{d} w_{1} \mathrm{~d} w_{2} \mathrm{~d} w_{3}\left\langle\psi\left(w_{1}\right) \psi\left(w_{2}\right) \psi\left(w_{3}\right)\right\rangle_{\mathbf{a}}^{c} \\
& +\lambda_{*} \epsilon^{2 h-2} \int \mathrm{d}^{2} u\langle\phi(u, \bar{u})\rangle_{\mathbf{a}}^{c}+\lambda_{*} \mu_{*} \epsilon^{3 h-3} \int \mathrm{d}^{2} u \mathrm{~d} w\langle\phi(u, \bar{u}) \psi(w)\rangle_{\mathbf{a}}^{c} \\
& \left.+\frac{1}{2} \lambda_{*} \mu_{*}^{2} \epsilon^{4 h-4} \int \mathrm{d}^{2} u \mathrm{~d} w_{1} \mathrm{~d} w_{2}\left\langle\phi(u, \bar{u}) \psi\left(w_{1}\right) \psi\left(w_{2}\right)\right\rangle_{\mathbf{a}}^{c}+\cdots\right) .
\end{aligned}
$$

Here the suffix $c$ at the correlators indicates that we are only considering the connected components; for the terms that we have written explicitly above, this only makes a difference for the last contribution.

In order to identify the fixed points, we need to evaluate the perturbed $g$-function up to order $1 / \mathrm{m}^{3}$. Since both $\lambda_{*}$ and $\mu_{*}$ are of order $1 / \mathrm{m}$, we only need to consider terms that are at most of cubic order in these coupling constants ${ }^{5}$. We have written out explicitly all such terms except those that are proportional to $\lambda^{2}$. The reason for this is that to order $1 / \mathrm{m}^{3}$, they turn out not to depend on the boundary labels $\left(a_{1}, a_{2}\right)$, and therefore will not contribute to the relative entropy at order $1 / \mathrm{m}^{3}$. This can be seen from considering the possible asymptotics of the respective correlators. For the correlator of two bulk fields in the $\lambda^{2}$ term, there are the asymptotic channels $\mathbf{i}, \mathbf{j}=(1,1),(1,3),(1,5)$, and we have

$$
\left.\left\langle\phi\left(u_{1}, \bar{u}_{1}\right) \phi\left(u_{1}, \bar{u}_{1}\right)\right\rangle_{\mathbf{a}} \sim \sum_{\mathbf{i}, \mathbf{j}} B_{(1,3)} \mathbf{i} B_{(1,3)}\right)^{\mathbf{j}} D_{\mathbf{i j}}^{(1,1)}\langle\mathbb{1}\rangle_{\mathbf{a}} f_{\mathbf{i j}}\left(u_{1}, \bar{u}_{1}, u_{2}, \bar{u}_{2}\right),
$$

where the $f_{\mathrm{ij}}$ are some functions that give the asymptotic dependence on the insertion points, compare (2.15). With the OPE constants from appendix A.1, one can see that all channels contribute boundary-dependent terms only at order $1 / \mathrm{m}^{2}$, so that the whole contribution of the $\lambda^{2}$ term will be of order $1 / m^{4}$. For the terms with coupling constants $\lambda^{2} \mu$ and $\lambda^{3}$, a similar analysis shows that their contributions will only affect the order $1 / \mathrm{m}^{4}$ as well.

The above integrals are not well defined, and we need to introduce a regularization scheme to make sense of them. In each case the leading term in $1 / m$ that is dependent on the boundary labels $\left(a_{1}, a_{2}\right)$ turns out to be of order $1 / \mathrm{m}^{3}$, and thus we are effectively working to leading order in $1 / \mathrm{m}$. In particular, we can therefore take the large $m$ expansion of the integrand before we do the integral. This integral will be regularized by some cut-off scheme; in particular, we shall introduce a cut-off $\epsilon$ to separate the boundary fields from one another, and a cut-off $\xi$ to restrict the radial bulk integration from $0 \leqslant r \leqslant 1-\xi$. We shall then discard the terms proportional to $\epsilon^{-1}$, as they describe non-universal terms (that have the wrong scaling behaviour). We shall also impose a similar procedure for the terms that are singular in $\xi \rightarrow 0$ which we shall describe in more detail below.

We should note that this cut-off regularization scheme is obviously not the same as the scheme with which the RG equations of section 2 were derived. However, to leading order in $1 / m$ the quantities we calculate should be universal, and thus this distinction should not play a role; this expectation will be borne out by our results. We have also checked this explicitly for some of the terms; the advantage of the cut-off scheme we are using here is that

5 To leading order in $1 / \mathrm{m}$, the bare and the renormalized coupling constants agree, and we we can therefore directly use the above fixed points (for the renormalized coupling constants) here. 
the calculations are much simpler since we do not need to know the integrand for arbitrary $m$ (but only in the $m \rightarrow \infty$ limit).

We shall now discuss the various terms in turn. The first two terms are the pure boundary integrals that were already considered in [24]. The boundary contribution proportional to $\mu_{*}^{2}$ involves the integral

$$
\begin{aligned}
I_{1} & =\int \mathrm{d} \theta_{1} \mathrm{~d} \theta_{2}\left|2 \sin \frac{\theta_{1}-\theta_{2}}{2}\right|^{-2 h}=\frac{\pi}{2} \int_{\epsilon}^{2 \pi-\epsilon} \mathrm{d} \theta\left(\sin ^{-2} \frac{\theta}{2}+\frac{4 \log \left(2 \sin \frac{\theta}{2}\right)}{\sin ^{2} \frac{\theta}{2}} \frac{1}{m}+\mathcal{O}\left(m^{-2}\right)\right) \\
& =2 \pi\left(\cot \frac{\epsilon}{2}+\frac{2}{m}\left(\epsilon-\pi+\cot \frac{\epsilon}{2}\left(1+\log \left(2 \sin \frac{\epsilon}{2}\right)\right)\right)\right)+\mathcal{O}\left(m^{-2}\right) \\
& =\frac{4 \pi}{\epsilon}+\left(\frac{16 \pi(1+\log \epsilon)}{\epsilon}-4 \pi^{2}\right) \frac{1}{m}+\mathcal{O}\left(\epsilon, m^{-2}\right)
\end{aligned}
$$

The first nontrivial contribution of the integral is hence of order $1 / m$, and we obtain the contribution (dropping the non-universal term proportional to $1 / \epsilon$ )

$$
\begin{aligned}
\frac{1}{2} \mu_{*}^{2} \epsilon^{2 h-2} \int \mathrm{d} w_{1} \mathrm{~d} w_{2}\left\langle\psi\left(w_{1}\right) \psi\left(w_{2}\right)\right\rangle_{\mathbf{a}}^{c} & =-\pi^{2} \frac{2}{m} D_{(1,3)(1,3)}^{(1,1)}\langle\mathbb{1}\rangle_{\mathbf{a}} \mu_{*}^{2}+\mathcal{O}\left(m^{-4}\right) \\
& =-\frac{4 \pi^{2}}{m}\left(\frac{\mu_{*}}{\alpha}\right)^{2}\langle\mathbb{1}\rangle_{\mathbf{a}}\left(a_{2}^{2}-1\right)+\mathcal{O}\left(m^{-4}\right)
\end{aligned}
$$

where the correction term is of order $m^{-4}$ for all fixed points of interest. The boundary contribution depending on $\mu_{*}^{3}$ involves the integral

$$
\begin{aligned}
I_{2} & =\int \mathrm{d} \theta_{1} \mathrm{~d} \theta_{2} \mathrm{~d} \theta_{3}\left|8 \sin \frac{\theta_{12}}{2} \sin \frac{\theta_{23}}{2} \sin \frac{\theta_{31}}{2}\right|^{-h} \\
& =4 \pi \int_{\theta_{1}>\theta_{2}} \mathrm{~d} \theta_{1} \mathrm{~d} \theta_{2}\left(8 \sin \frac{\theta_{12}}{2} \sin \frac{\theta_{1}}{2} \sin \frac{\theta_{2}}{2}\right)^{-1}+\mathcal{O}\left(m^{-1}\right) .
\end{aligned}
$$

If we introduce cut-offs only where necessary, so that the integral does not diverge, we find

$$
I_{2}=8 \pi \cot \frac{\epsilon}{2}-4 \pi^{2}+\mathcal{O}\left(m^{-1}\right)=\frac{16 \pi}{\epsilon}-4 \pi^{2}+\mathcal{O}\left(\epsilon, m^{-1}\right)
$$

Again dropping the non-universal $1 / \epsilon$ term, we get the contribution

$$
\begin{aligned}
\frac{1}{6} \mu_{*}^{3} \epsilon^{3 h-3} \int \mathrm{d} w_{1} \mathrm{~d} w_{2} \mathrm{~d} w_{3}\left\langle\psi\left(w_{1}\right) \psi\left(w_{2}\right) \psi\left(w_{3}\right)\right\rangle_{\mathbf{a}}^{c} \\
=\frac{2 \pi^{2}}{3} D_{(1,3)(1,3)} D_{(1,3)(1,3)}\langle\mathbb{1}\rangle_{\mathbf{a}} \mu_{*}^{3}+\mathcal{O}\left(m^{-4}\right) \\
=\frac{16 \pi^{2}}{3}\left(\frac{\mu_{*}}{\alpha}\right)^{3}\langle\mathbb{1}\rangle_{\mathbf{a}}\left(a_{2}^{2}-1\right)+\mathcal{O}\left(m^{-4}\right)
\end{aligned}
$$

The contribution which depends linearly on $\lambda_{*}$ and is independent of $\mu_{*}$ involves the integral

This yields

$$
\begin{aligned}
I_{3} & =\int \mathrm{d} r r\left(1-r^{2}\right)^{-2 h}=\int_{0}^{1-\xi} \mathrm{d} r r\left(1-r^{2}\right)^{-2}+\mathcal{O}\left(m^{-1}\right) \\
& =\frac{(1-\xi)^{2}}{2 \xi(2-\xi)}+\mathcal{O}\left(m^{-1}\right)=\frac{1}{8} \frac{2-3 \xi}{\xi}+\mathcal{O}\left(\xi, m^{-1}\right)
\end{aligned}
$$

$$
\begin{aligned}
\lambda_{*} \epsilon^{2 h-2} \int \mathrm{d}^{2} u\langle\phi(u, \bar{u})\rangle_{\mathbf{a}}^{c} & =\lambda_{*}\langle\mathbb{1}\rangle_{\mathbf{a}} B_{(1,3)}^{(1,1)}\left(\frac{\pi}{4} \frac{2-3 \xi}{\xi}+\mathcal{O}\left(\xi, m^{-1}\right)\right) \\
& =\lambda_{*}\langle\mathbb{1}\rangle_{\mathbf{a}}\left(-\frac{\pi^{3}\left(a_{2}^{2}-1\right)}{m^{2}} \frac{2-3 \xi}{\xi}+f(m, \xi)+\mathcal{O}\left(\xi, m^{-1}\right)\right),
\end{aligned}
$$


where $f(m, \xi)$ is some function which does not depend on the boundary labels and has the large- $m$ asymptotic behaviour

$$
f(m, \xi)=\frac{3 \pi}{4} \frac{2-3 \xi}{\xi}+\mathcal{O}\left(\xi, m^{-1}\right)
$$

The contribution that depends linearly on $\lambda_{*}$ and $\mu_{*}$ involves the integral

$$
\begin{aligned}
I_{4} & =\int \mathrm{d} r \mathrm{~d} \theta_{1} \mathrm{~d} \theta_{2} r\left(\left(1-r^{2}\right)\left(1-2 r \cos \left(\theta_{1}-\theta_{2}\right)+r^{2}\right)\right)^{-h} \\
& =\int_{0}^{1-\xi} \mathrm{d} r \frac{4 \pi^{2} r}{\left(1-r^{2}\right)^{2}}+\mathcal{O}\left(m^{-1}\right)=\frac{\pi^{2}}{2} \frac{2-3 \xi}{\xi}+\mathcal{O}\left(\xi, m^{-1}\right),
\end{aligned}
$$

leading to

$$
\begin{aligned}
\lambda_{*} \mu_{*} \epsilon^{3 h-3} \int & \mathrm{d}^{2} u \mathrm{~d} w\langle\phi(u, \bar{u}) \psi(w)\rangle_{\mathbf{a}}^{c} \\
= & \lambda_{*} \mu_{*}\langle\mathbb{1}\rangle_{\mathbf{a}} B_{(1,3)}^{(1,3)} D_{(1,3)(1,3)}^{(1,1)}\left(\frac{\pi^{2}}{2} \frac{2-3 \xi}{\xi}+\mathcal{O}\left(\xi, m^{-1}\right)\right) \\
= & \lambda_{*}\left(\frac{\mu_{*}}{\alpha}\right)\langle\mathbb{1}\rangle_{\mathbf{a}} \frac{4 \pi^{3}}{m}\left(a_{2}^{2}-1\right)\left(\frac{2-3 \xi}{\xi}+\mathcal{O}\left(\xi, m^{-1}\right)\right) .
\end{aligned}
$$

Finally, the integrand for the term of order $\lambda_{*} \mu_{*}^{2}$ is the same as in the calculation of the coefficient $E$ in the RG equation of section 2. Deferring the details of this calculation to appendix $\mathrm{B}$, the resulting contribution turns out to be

$$
\begin{aligned}
\frac{1}{2} \lambda_{*} \mu_{*}^{2} \epsilon^{4 h-4} \int & \mathrm{d}^{2} u \mathrm{~d} w_{1} \mathrm{~d} w_{2}\left\langle\phi(u, \bar{u}) \psi\left(w_{1}\right) \psi\left(w_{2}\right)\right\rangle_{\mathbf{a}}^{c} \\
= & \frac{\pi}{3} \lambda_{*} \mu_{*}^{2}\langle\mathbb{1}\rangle_{\mathbf{a}} B_{(1,3)}^{(1,1)} D_{(1,1)(1,3)}^{(1,3)} D_{(1,3)(1,3)}^{(1,1)}\left(-2 \pi^{2} \frac{2-3 \xi}{\xi}+\mathcal{O}(\xi)+\cdots\right) \\
= & -\lambda_{*}\left(\frac{\mu_{*}}{\alpha}\right)^{2}\langle\mathbb{1}\rangle_{\mathbf{a}} 4 \pi^{3}\left(a_{2}^{2}-1\right) \frac{2-3 \xi}{\xi}+\mathcal{O}\left(\xi, m^{-4}\right),
\end{aligned}
$$

where the ellipses in the second line refer to terms of order $\mathcal{O}\left(\epsilon, m^{-1}\right)$. Adding all the relevant terms together, we then arrive at

$$
\begin{aligned}
\log \left\langle\mathrm{e}^{\delta S}\right\rangle_{\mathbf{a}}= & \log \langle\mathbb{1}\rangle_{\mathbf{a}}+\left(a_{2}^{2}-1\right)\left[-\frac{4 \pi^{2}}{m}\left(\frac{\mu_{*}}{\alpha}\right)^{2}+\frac{16 \pi^{2}}{3}\left(\frac{\mu_{*}}{\alpha}\right)^{3}\right. \\
& \left.+\lambda_{*} \frac{2-3 \xi}{\xi}\left(-\frac{\pi^{3}}{m^{2}}+\frac{4 \pi^{3}}{m}\left(\frac{\mu_{*}}{\alpha}\right)-4 \pi^{3}\left(\frac{\mu_{*}}{\alpha}\right)^{2}\right)\right]+\cdots,
\end{aligned}
$$

where the ellipses either denote terms that are independent of the boundary labels or terms that are of order $\mathcal{O}\left(\mathrm{m}^{-4}\right)$ for the fixed points of interest. The first two coefficients (that are independent of $\lambda_{*}$ ) reproduce exactly what was found in [24]. Using their results, it thus follows that the fixed point (I) is indeed the perturbative fixed point of the pure boundary perturbation.

3.1.1. Fixed point (II). At the fixed point (II), we have $\frac{\mu_{*}}{\alpha}=\frac{1}{2 m}$, and it follows from (3.17) that the last three terms (that are all proportional to $\lambda_{*}$ ) cancel identically. The perturbed $g$ function (3.17) is then given by

$$
\log g_{\lambda_{*}, \mu_{*}}^{(m)}(\mathbf{a})=\log g^{(m)}(\mathbf{a})-\frac{\pi^{2}}{3 m^{3}}\left(a_{2}^{2}-1\right)+f(m)+\mathcal{O}\left(m^{-4}\right),
$$


where $f(m)$ is a function which is at least of order $1 / m$ and does not depend on the boundary labels. Subtracting the corresponding expression for the boundary entropy of the $(1,1)$ boundary condition, we thus find that the perturbed relative entropy $\hat{g}$ equals

$$
\begin{aligned}
\hat{g}_{\lambda_{*}, \mu_{*}}^{(m)}(\mathbf{a}) & =\hat{g}^{(m)}(\mathbf{a})\left(1-\frac{\pi^{2}}{3}\left(a_{2}^{2}-1\right) \frac{1}{m^{3}}+\mathcal{O}\left(m^{-4}\right)\right) \\
& =a_{1} a_{2}\left(1-\frac{\pi^{2}}{6}\left(a_{1}^{2}+a_{2}^{2}-2\right) \frac{1}{m^{2}}+\mathcal{O}\left(m^{-4}\right)\right),
\end{aligned}
$$

where in the final line we have used the asymptotic expansion (3.3) for $\hat{g}^{(m)}(\mathbf{a})$.

This is now to be compared with the relative entropy of the boundary condition $\mathbf{b}=\left(b_{1}, b_{2}\right)$ in the $c_{m-1}$ theory:

$\hat{g}^{(m-1)}(\mathbf{b})=b_{1} b_{2}\left(1-\frac{\pi^{2}}{6}\left(b_{1}^{2}+b_{2}^{2}-2\right) \frac{1}{m^{2}}-\frac{\pi^{2}}{3}\left(b_{1}^{2}-1\right) \frac{1}{m^{3}}+\mathcal{O}\left(m^{-4}\right)\right)$.

If $a_{1}=1$, (3.20) equals (3.19) for a single fundamental boundary condition $\mathbf{b}$ :

$$
\mathbf{a}=\left(1, a_{2}\right)_{m} \stackrel{(\mathrm{II})}{\longrightarrow} \mathbf{b}=\left(1, a_{2}\right)_{m-1}
$$

In the general case, we cannot solve for $(3.19)=(3.20)$ with a single boundary condition $\mathbf{b}=\left(b_{1}, b_{2}\right) .{ }^{6}$ As in [24], we therefore consider superpositions of fundamental boundary conditions

$$
\mathbf{B}=\bigoplus_{l=1}^{N} \mathbf{b}^{l}, \quad \mathbf{b}^{l}=\left(b_{1}^{l}, b_{2}^{l}\right)
$$

The entropy of the superposition is just the sum of the individual entropies, and we thus get the equations

$$
\begin{aligned}
& \sum_{l} b_{1}^{l} b_{2}^{l}=a_{1} a_{2}, \\
& \sum_{l} b_{1}^{l} b_{2}^{l}\left(\left(b_{1}^{l}\right)^{2}+\left(b_{2}^{l}\right)^{2}-2\right)=a_{1} a_{2}\left(a_{1}^{2}+a_{2}^{2}-2\right), \\
& \sum_{l} b_{1}^{l} b_{2}^{l}\left(\left(b_{1}^{l}\right)^{2}-1\right)=0 .
\end{aligned}
$$

The last equation implies that $b_{1}^{l}=1$ for all $l$, and the equations are generically solved by

$$
N=\min \left(a_{1}, a_{2}\right), \quad b_{1}^{l}=1 \quad \forall l, \quad b_{2}^{l}=a_{1}+a_{2}+1-2 l .
$$

This suggests flows of the form

$$
\mathbf{a}=\left(a_{1}, a_{2}\right)_{m} \stackrel{(\mathrm{II})}{\longrightarrow} \mathbf{B}=\sum_{l=1}^{\min \left(a_{1}, a_{2}\right)}\left(1, a_{1}+a_{2}+1-2 l\right)_{m-1} .
$$

These flows have to be understood as sequences of flows (first a pure boundary flow to (I) followed by the flow to (II)), as was discussed at the end of section 2 (see also figure 1). Note that the result of (3.25) is similar to what happened in the case of a pure boundary perturbation [24], except that there the endpoints of the boundary flow were superpositions of boundary conditions $\left(b_{l}, 1\right)$, while here we have boundary conditions $\left(1, b_{l}\right)$.

6 In [44], a similar calculation was done to lower order in $1 / m$, from which the authors concluded that the flow is simply $\left(a_{1}, a_{2}\right) \rightarrow\left(a_{2}, a_{1}\right)$. This is compatible with the analysis to order $1 / \mathrm{m}^{2}$, but not to order $1 / \mathrm{m}^{3}$. 
We should mention that the $g$ function does not allow us to determine the resulting boundary conditions uniquely, since

$$
\hat{g}^{(m-1)}\left(b_{1}, b_{2}\right)=\hat{g}^{(m-1)}\left(b_{1}, m-b_{2}\right) .
$$

One can partially fix this ambiguity as in [24]. To zeroth order in perturbation theory, we know that the $(r, s)$ bulk field of the $m$ th theory becomes the $(s, r)$ bulk field of the $(m-1)$ th theory [1]. Thus to leading order in $1 / m$ we need to have that

$$
\left({ }^{\mathbf{a}} B_{(r, s)}^{(1,1)}\right)^{(m)}=\left({ }^{\mathbf{B}} B_{(s, r)}^{(1,1)}\right)^{(m-1)} .
$$

Since

$$
\left({ }^{\left(a_{1}, a_{2}\right)} B_{(r, s)}^{(1,1)}\right)^{(m)}=(-1)^{(r+s)\left(a_{1}+a_{2}\right)} \frac{\sin \left(\frac{r a_{1} \pi}{m}\right) \sin \left(\frac{s a_{2} \pi}{m+1}\right)}{\sin \left(\frac{a_{1} \pi}{m}\right) \sin \left(\frac{a_{2} \pi}{m+1}\right)},
$$

this requires, in particular, that

$$
(-1)^{(r+s)\left(a_{1}+a_{2}\right)}=(-1)^{(r+s)\left(b_{1}^{l}+b_{2}^{l}\right)}
$$

for all $(r, s)$ and all $l$. This is evidently satisfied by our ansatz, but would not in general be true if we replaced some $\left(b_{1}^{l}, b_{2}^{l}\right)$ by $\left(b_{1}^{l}, m-b_{2}^{l}\right)$.

3.1.2. Fixed point (III). The analysis for the fixed point (III) is more complicated, since now the $\lambda_{*}$ term in the last line of (3.17) does contribute. We therefore need to understand how to deal with the singular part as $\xi \rightarrow 0$. We do not have a fundamental understanding of how this must be done, but we shall now propose a procedure that will lead to consistent results. In fact, the procedure is already determined by considering the consistency of the flow of the $\left(a_{1}, 1\right)$ boundary condition (see section 4 , as well as the remarks below in section 3.2), and the fact that all the other consistency conditions then also work out (that will be explained in detail in section 4) makes us confident that this is indeed the correct prescription ${ }^{7}$.

Since the cut-off $\xi$ bounds the radial bulk integral to the region $0 \leqslant r \leqslant 1-\xi$, it is actually a measure of an area; in fact, the area of the missing annulus is simply $\pi\left(1-(1-\xi)^{2}\right)=2 \pi \xi(1-\xi / 2)$. In order to convert it to a parameter that scales as a length, we should divide it by a natural length, which we take to be the circumference of the inner circle, i.e. $2 \pi(1-\xi)$. We thus propose that the good cut-off parameter is

$$
\eta=\frac{\xi(1-\xi / 2)}{(1-\xi)}=\xi(1+\xi / 2)+\mathcal{O}\left(\xi^{3}\right) .
$$

The expression of interest can therefore be written as

$$
\frac{2-3 \xi}{\xi}=\frac{2}{\eta}-2+\mathcal{O}(\eta)
$$

Our prescription is now that we should discard the non-universal $1 / \eta$ pole, but keep the constant term. Using that at (III) $\frac{\mu_{*}}{\alpha}=\frac{1}{m}$ as well as $\lambda_{*}=-\frac{1}{\pi m}$ then leads to

$$
\log g_{\lambda_{*}, \mu_{*}}^{(m)}(\mathbf{a})=\log g^{(m)}(\mathbf{a})-\frac{2 \pi^{2}}{3 m^{3}}\left(a_{2}^{2}-1\right)+f(m)+\mathcal{O}\left(m^{-4}\right) .
$$

The perturbed relative entropy $\hat{g}$ then equals

$$
\begin{aligned}
\hat{g}_{\lambda_{*}, \mu_{*}}^{(m)}(\mathbf{a}) & =\hat{g}^{(m)}(\mathbf{a})\left(1-\frac{2 \pi^{2}}{3}\left(a_{2}^{2}-1\right) \frac{1}{m^{3}}+\mathcal{O}\left(m^{-4}\right)\right) \\
& =a_{1} a_{2}\left(1-\frac{\pi^{2}}{6}\left(a_{1}^{2}+a_{2}^{2}-2\right) \frac{1}{m^{2}}-\frac{\pi^{2}}{3 m^{3}}\left(a_{2}^{2}-1\right)+\mathcal{O}\left(m^{-4}\right)\right),
\end{aligned}
$$

7 The prescription we propose actually has a very natural interpretation if we consider the theory on the semi-infinite cylinder (see appendix B.2). We thank Anatoly Konechny for discussions on this point. 
where in the final line we have used the asymptotic expansion (3.3) for $\hat{g}^{(m)}(\mathbf{a})$. Comparing this with (3.20) we then find that the endpoint should be given by

$$
\left(a_{1}, a_{2}\right)_{m} \stackrel{\text { (III) }}{\longrightarrow}\left(a_{2}, a_{1}\right)_{m-1} .
$$

This flow has to be understood as a sequence of asymptotic flows, similar to (3.25).

\subsection{The analysis for $a_{2}=1$}

For $a_{2}=1$, the analysis is simpler since the boundary condition does not have the relevant boundary field $\psi_{(1,3)}$ in its spectrum, and thus we have no RG equation for $\mu$. Of the terms in (3.4) hence only the term (3.12) survives. However, since it does not depend on $a_{1}$, and since we are only interested in the ratio of the perturbed $g$-function relative to $\mathbf{a}=(1,1)$, it does not contribute to the rescaled $g$ function in the $(m-1)$ th theory. Thus we conclude that the (rescaled) $g$-function does not change at all. Using equation (3.1), we find that

$$
\hat{g}^{(m)}\left(a_{1}, 1\right)=\frac{\sin \frac{\pi a_{1}}{m}}{\sin \frac{\pi}{m}}=\hat{g}^{(m-1)}\left(1, a_{1}\right) .
$$

This then establishes (2.25). Note that this argument holds for arbitrary values of $a_{1}$, not necessarily small relative to $m$.

Actually, since in this case no combined flow takes place, one may suspect that not only is the ratio of $g$-functions correctly reproduced by this analysis but also the overall value of the $g$-function. As in the pure boundary case, let us consider the logarithmic change of the $g$ function. The only term that contributes in this case is the term (3.12) that contains a contribution of order $1 / m$ that is independent of the boundary labels. Using (3.13), we thus have to first order

$$
\log \frac{g_{\lambda_{*}, \mu_{*}}^{(m)}\left(a_{1}, 1\right)}{g^{(m)}\left(a_{1}, 1\right)}=-\frac{3}{4 m} \frac{2-3 \xi}{\xi}+\mathcal{O}\left(\xi, m^{-2}\right),
$$

which we must compare with the expansion of the resulting boundary condition,

$$
\log \frac{g^{(m-1)}\left(1, a_{1}\right)}{g^{(m)}\left(a_{1}, 1\right)}=\frac{3}{2 m}+\mathcal{O}\left(m^{-2}\right) .
$$

These two expressions then agree precisely to this order if we use the same prescription as in (3.31).

\section{The actual fixed point and consistency constraints}

So far we have identified the perturbative fixed points by studying the perturbed $g$-function. The actual fixed point (IV) of the RG flow for a generic initial condition however appears at $\lambda_{*}=-\frac{1}{\pi m}$ and $\mu_{*}=+\infty$ (for $\alpha<0$-for $\alpha>0$ we have instead $\mu_{*}=-\infty$ ), as is clear from the flow diagram (see figure 1). We now want to combine what we have found so far with results about non-perturbative boundary flows in order to identify this actual (non-perturbative) fixed point. In order to tie it down completely, we shall also use some constraints that arise upon using defects.

First of all, one way to get to (IV) is to turn on a $\psi_{(1,3)}$ field on the boundary condition (II). Indeed, as we have shown above in (2.26), the coupling constant $\tilde{\mu}$ couples to a $\psi_{(1,3)}$ field at the fixed point (II). Furthermore, the flow from (II) to (III) is the usual perturbative $\psi_{(1,3)}$ flow, and thus the flow from (II) to (IV) must be the corresponding non-perturbative flow (where the field $\psi_{(1,3)}$ is switched on with the opposite sign). The situation is particularly simple if the boundary spectrum of (II) only contains a single $\psi_{(1,3)}$ field-then it is clear that this is the 
field to which $\tilde{\mu}$ must couple. This will be the case provided that we begin with a boundary condition of type $\left(1, a_{2}\right)_{m}$, since then the perturbative fixed point (II) consists of a single boundary condition $\left(1, a_{2}\right)_{m-1}$ that has a single $\psi_{(1,3)}$ boundary field in its spectrum. The fixed point (III) is then the endpoint $\left(a_{2}, 1\right)_{m-1}$ of the perturbative boundary flow (provided that $a_{2}$ is not too large) [24].

In this case we can determine the endpoint of the non-perturbative $\psi_{(1,3)}$ flow, using the results of $[16,27,30]$ that have been obtained using TCSA and TBA techniques (see also $[34,35])$. For small label $a_{2}$, the result is simply

$$
\left(1, a_{2}\right)_{m-1} \longrightarrow\left(a_{2}-1,1\right)_{m-1}
$$

Summarizing our results so far, we predict the endpoints of the RG flows to be

$$
\begin{aligned}
& \left(a_{1}, 1\right)_{m} \longrightarrow\left(1, a_{1}\right)_{m-1} \\
& \left(1, a_{2}\right)_{m} \longrightarrow\left(a_{2}-1,1\right)_{m-1} \quad \text { for } \quad 1<a_{2} \ll m .
\end{aligned}
$$

If we now want to consider the case of a general boundary condition $\left(a_{1}, a_{2}\right)_{m}$, we face the problem that we do not know a priori how to identify the boundary field that couples to $\tilde{\mu}$. One way to proceed would be to argue that the flow in question must be the non-perturbative flow corresponding to the perturbative flow from (II) to (III) that we have identified above; this will, as we shall see, lead to the correct result. However, in the following we shall follow a different route by studying the action of topological defect lines.

\subsection{Defect lines}

So far we have discussed how boundary conditions adjust themselves under bulk deformations. We can also use similar methods to analyse what happens to defect lines. Recall that defect lines interpolate between in general different conformal field theories. In the following, we shall only consider defects where the theories on both sides are the same (namely some minimal model). More specifically, we shall consider topological defects that are characterized by the property that the corresponding defect operator commutes with the action of both left- and right-moving Virasoro generators. In particular, this implies that the defect only depends on the homotopy class of the defect line [36]. By moving the defect line to the boundary (i.e. by 'fusion'), such topological defects then define an action on the (conformal) boundary conditions of the theory.

For the charge conjugation theories we are considering here, the topological defects are labelled by the same labels as the Cardy boundary states. We can therefore denote them as $\mathcal{D}\left(d_{1}, d_{2}\right)$, where $\mathbf{d}=\left(d_{1}, d_{2}\right)$ has the same range and identification rules as the boundary labels $\mathbf{a}=\left(a_{1}, a_{2}\right)$. The action of the defect $\mathcal{D}(\mathbf{d})$ on the boundary condition $\mathbf{a}$ is then described by the usual fusion rules

$$
\mathcal{D}(\mathbf{d}) \times \mathbf{a}=\bigoplus_{\mathbf{b}} N_{\mathbf{d a}}^{\mathbf{b}} \mathbf{b} .
$$

In addition to the identity defect $\mathcal{D}(1,1)$, there is a special topological defect $\mathcal{D}(m-1,1) \cong$ $\mathcal{D}(1, m)$ that generates a $\mathbb{Z}_{2}$ symmetry of the charge conjugation minimal model (such a defect is called a 'group-like defect' in [37] and a 'symmetry defect' in [38]). Its action on a bulk field $\phi_{(r, s)}$ is given by

$$
\mathcal{D}(1, m): \quad \phi_{(r, s)} \mapsto(-1)^{(m+1) r-m s+1} \phi_{(r, s)},
$$


which is indeed a symmetry of the bulk theory. Note that the field $\phi_{(1,3)}$ that we use to perturb the theory is invariant under this $\mathbb{Z}_{2}$ action. The effect of the $\mathbb{Z}_{2}$ symmetry on a boundary condition is

$$
\left(a_{1}, a_{2}\right) \mapsto \mathcal{D}(1, m) \times\left(a_{1}, a_{2}\right)=\left(a_{1}, m+1-a_{2}\right) .
$$

The concept of defect lines has proven very useful in the discussion of boundary flows $[39,40]$ and combined bulk-boundary flows [38].

After these preparations, we now want to study what happens to these topological defects under the bulk deformation where we perturb the bulk theory on both sides of the defect by the $\phi_{(1,3)}$ bulk field. In principle, this can also be analysed by the above methods since we can think of the defect as a boundary condition in the doubled theory [41]. Typically, the bulk deformation will switch on defect fields - the analogue of the boundary fields for boundary conditions-that will ensure that at the end of the flow the defect is again conformal. In general, however, a topological defect will not flow to a topological defect again, and thus the identification of the endpoint will be difficult.

There is, however, one particularly simple class of defects for which the analysis is essentially trivial. These are the defects $\mathcal{D}\left(d_{1}, 1\right)$, on which no relevant defect operator can be turned on by the $\phi_{(1,3)}$ perturbation. In fact, the spectrum of a topological defect $\mathcal{D}(\mathbf{d})$ is given by

$$
\mathcal{H}_{\mathcal{D}(\mathbf{d})}=\bigoplus_{\mathbf{a}, \mathbf{b}, \mathbf{c}} N_{\mathbf{d c}}{ }^{\mathbf{d}} N_{\mathbf{a b}} \mathbf{c}^{\mathbf{c}} \mathcal{H}_{\mathbf{a}} \otimes \mathcal{H}_{\mathbf{b}}
$$

The defect spectrum can be decomposed under the action of the two Virasoro algebras. Thus each defect field $\psi_{\mathbf{a b}}$ carries two labels $\mathbf{a}=\left(a_{1}, a_{2}\right)$ and $\mathbf{b}=\left(b_{1}, b_{2}\right)$. For $\mathbf{d}=\left(d_{1}, 1\right)$, we only find fields in the spectrum for which $\mathbf{b}=\left(a_{1}+2 n, a_{2}\right)$ for some integer $n$. As the bulk field $\phi_{(1,3)}$ can only turn on defect fields with $a_{1}=b_{1}=1$ and $a_{2}, b_{2}$ odd, we can only turn on the defect fields $\psi_{(1,1+2 p)(1,1+2 p)}$ (for some integer $p$ ) on $\mathcal{D}\left(d_{1}, 1\right)$. Apart from the identity field, the field with the lowest conformal weight is $\psi_{(1,3)(1,3)}$ whose conformal weight is $h=2 h_{(1,3)}=1+\frac{m-3}{m+1}$. This is greater than 1 for $m>3$, and thus the field is irrelevant.

This argument shows that no relevant defect fields are turned on in our case. This should then imply that the endpoint of the flow is again a topological defect ${ }^{8}$. Under this assumption it is then easy to determine to which topological defect $\mathcal{D}\left(d_{1}, 1\right)_{m}$ flows in the $(m-1)$ th model. To see how this goes, let us consider a configuration with a defect line $\mathcal{D}\left(d_{1}, 1\right)_{m}$ and the simplest boundary condition $(1,1)_{m}$. Now we can either first act with the defect line on the boundary and then do the bulk perturbation, or we can first analyse the bulk perturbation when the defect and the boundary are far apart, and then let the perturbed defect act on the perturbed boundary condition. If we first let the defect act on the boundary condition in the $m$ th theory, we get the boundary condition $\left(d_{1}, 1\right)_{m}$, which under the bulk perturbation flows to $\left(1, d_{1}\right)_{m-1}$ (see (4.2)). If we do the bulk perturbation first, we flow to a topological defect $\tilde{D}_{m-1}$ and the boundary condition $(1,1)_{m-1}$. To have compatibility with the above discussion, the action of the (topological) defect $\tilde{D}_{m-1}$ on $(1,1)_{m-1}$ must give $\left(1, d_{1}\right)_{m-1}$; the only topological defect that has this property is $\tilde{\mathcal{D}}_{m-1}=\mathcal{D}\left(1, d_{1}\right)_{m-1}$. Thus, we conclude that

$$
\mathcal{D}\left(d_{1}, 1\right)_{m} \longrightarrow \mathcal{D}\left(1, d_{1}\right)_{m-1} \text {. }
$$

Note that this rule should be true for all values of $d_{1}$ since (4.2) holds for all values of $a_{1}$. In particular, it also applies to the defect $\mathcal{D}(m-1,1)_{m}$ that generates the $\mathbb{Z}_{2}$ symmetry. As the bulk perturbation by $\phi_{(1,3)}$ is invariant under the $\mathbb{Z}_{2}$ symmetry, the defect $\mathcal{D}(m-1,1)_{m}$ should

8 An independent argument for this defect to remain topological under the renormalization group flow was also very recently given in [42]. 
flow to the $\mathbb{Z}_{2}$ generating defect $\mathcal{D}(m-2,1)_{m-1} \cong \mathcal{D}(1, m-1)_{m-1}$ in the $(m-1)$ th theory, which is precisely consistent with (4.8).

As another consistency check of this proposal, we can consider the action on bulk fields. From the analysis of [1], we know that the $(r, s)$ bulk field in the $m$ th theory flows to a linear combination $\phi^{\prime}$ of bulk fields

$$
\phi_{(r, s)}^{(m)} \longrightarrow \phi^{\prime}=\sum_{s^{\prime}-s \text { even }} c_{s^{\prime}}\left[\phi_{\left(s^{\prime}, r\right)}^{(m-1)}\right]
$$

where $c_{s^{\prime}}$ are some constants and we indicated by the square brackets that also descendants of the primary bulk fields can appear. On the other hand, we know the action of a topological defect $\mathcal{D}\left(d_{1}, d_{2}\right)_{m}$ on a bulk field in the $m$ th theory (see e.g. [36]):

$$
\mathcal{D}\left(d_{1}, d_{2}\right)_{m} \phi_{(r, s)}^{(m)}=(-1)^{\left(d_{1}-d_{2}\right)(r-s)} \frac{\sin \left(\frac{\pi d_{1} r}{m}\right)}{\sin \left(\frac{\pi r}{m}\right)} \frac{\sin \left(\frac{\pi d_{2} s}{m+1}\right)}{\sin \left(\frac{\pi s}{m+1}\right)} \phi_{(r, s)}^{(m)} .
$$

If we first apply the defect $\mathcal{D}\left(d_{1}, 1\right)_{m}$ in the $m$ th theory, and then flow to the $(m-1)$ th theory, we obtain

$\mathcal{D}\left(d_{1}, 1\right)_{m} \phi_{(r, s)}^{(m)}=(-1)^{\left(d_{1}-1\right)(r-s)} \frac{\sin \left(\frac{\pi d_{1} r}{m}\right)}{\sin \left(\frac{\pi r}{m}\right)} \phi_{(r, s)}^{(m)} \longrightarrow(-1)^{\left(d_{1}-1\right)(r-s)} \frac{\sin \left(\frac{\pi d_{1} r}{m}\right)}{\sin \left(\frac{\pi r}{m}\right)} \phi^{\prime}$.

On the other hand, if we first let both the defect and the bulk field flow, we have to evaluate

$$
\begin{aligned}
\mathcal{D}\left(1, d_{1}\right)_{m-1} \phi^{\prime} & =\sum_{s^{\prime}-s \text { even }} c_{s^{\prime}}(-1)^{\left(d_{1}-1\right)\left(r-s^{\prime}\right)} \frac{\sin \left(\frac{\pi d_{1} r}{m}\right)}{\sin \left(\frac{\pi r}{m}\right)}\left[\phi_{\left(s^{\prime}, r\right)}^{(m-1)}\right] \\
& =(-1)^{\left(d_{1}-1\right)(r-s)} \frac{\sin \left(\frac{\pi d_{1} r}{m}\right)}{\sin \left(\frac{\pi r}{m}\right)} \phi^{\prime},
\end{aligned}
$$

which precisely equals (4.11), thus giving further support to the proposal (4.8).

\subsection{The final answer}

Putting everything together, we can now determine what happens to an arbitrary boundary condition $\left(a_{1}, a_{2}\right)_{m}\left(1<a_{2} \ll m\right)$ under the bulk flow. To this end, we write it as the fusion of an appropriate topological defect on an elementary boundary condition and then perform the flow under the $\phi_{(1,3)}$ bulk perturbation,

$$
\begin{aligned}
\left(a_{1}, a_{2}\right)_{m} & =\mathcal{D}\left(a_{1}, 1\right)_{m} \times\left(1, a_{2}\right)_{m} \longrightarrow \mathcal{D}\left(1, a_{1}\right)_{m-1} \times\left(a_{2}-1,1\right)_{m-1} \\
& =\left(a_{2}-1, a_{1}\right)_{m-1} .
\end{aligned}
$$

Here we have assumed $1<a_{2} \ll m$ since we have used (4.3). On the other hand $a_{1}$ is arbitrary because the defect argument did not depend on $a_{1}$ being small or not. If $a_{2}=1$, there is no relevant boundary field in the boundary spectrum, and we have instead of (4.13) simply the flow (4.2).

Note that we can also use the defect argument to check our identifications for the perturbative fixed points (I), (II) and (III): if we apply the defects $\mathcal{D}\left(a_{1}, 1\right)_{m} \rightarrow \mathcal{D}\left(1, a_{1}\right)_{m-1}$ to the flow sequence for $a_{1}=1$,

$\left(1, a_{2}\right)_{m} \stackrel{\text { (I) }}{\longrightarrow}\left(a_{2}, 1\right)_{m} \stackrel{(\mathrm{II})}{\longrightarrow}\left(1, a_{2}\right)_{m-1} \stackrel{(\mathrm{III})}{\longrightarrow}\left(a_{2}, 1\right)_{m-1} \quad$ for $\quad 1<a_{2} \ll m$,

we find the sequence

$\left(a_{1}, a_{2}\right)_{m} \stackrel{(\mathrm{I})}{\longrightarrow} \bigoplus_{l}(b(l), 1)_{m} \stackrel{(\mathrm{II})}{\longrightarrow} \bigoplus_{l}(1, b(l))_{m-1} \stackrel{(\mathrm{IIII})}{\longrightarrow}\left(a_{2}, a_{1}\right)_{m-1} \quad$ for $\quad 1<a_{2} \ll m$, 
where $b(l)=\left|a_{1}-a_{2}\right|-1+2 l$ and $l=1, \cdots, \min \left(a_{1}, a_{2}, m-a_{1}, m-a_{2}\right)$. This reproduces in particular our results from section 2 for small values of $a_{1}$ and $a_{2}$. However, the current analysis is also true for arbitrary values of $a_{1}$ since the rule for the defect flow is not restricted to small values of $a_{1}$.

However, we still have the restriction that $a_{2}$ should be small. By using the identification rules $\left(a_{1}, a_{2}\right)_{m} \cong\left(m-a_{1}, m+1-a_{2}\right)_{m}$ we can also get a result for labels $a_{2}$ close to $m$. Firstly, we note that the flow (4.2) translates into

$$
\left(a_{1}, m\right)_{m} \longrightarrow\left(1, m-a_{1}\right)_{m-1} \text {. }
$$

For non-trivial label $a_{2}$, the sequence (4.15) of the perturbative fixed points (I), (II) and (III) is mapped to

$\left(a_{1}, a_{2}\right)_{m} \stackrel{(\mathrm{I})}{\longrightarrow} \bigoplus_{l}\left(b^{\prime}(l), 1\right)_{m} \stackrel{(\mathrm{II})}{\longrightarrow} \bigoplus_{l}\left(1, b^{\prime}(l)\right)_{m-1} \stackrel{(\mathrm{III})}{\longrightarrow}\left(a_{2}-2, a_{1}\right)_{m-1} \quad$ for $\quad 1 \ll a_{2}<m$,

where now $b^{\prime}(l)=\left|a_{1}-a_{2}+1\right|-1+2 l$ and $l=1, \cdots, \min \left(a_{1}, a_{2}-1, m-a_{1}, m+1-a_{2}\right)$. If we extrapolate this answer to small values of $a_{2}$, its form is different from that of the sequence (4.15). The reason for this is that the notion of which fixed points are perturbative and which are non-perturbative changes as we extrapolate $a_{2}$ from small to large values. In particular, instead of the perturbative pure boundary fixed point (2.19), the first flow (to fixed point (I)) is now of the same form as the non-perturbative boundary flow (that generalizes (4.1))

$$
\left(a_{1}, a_{2}\right) \longrightarrow \bigoplus_{l=1}^{\min \left(a_{1}, a_{2}-1\right)}\left(a_{1}+a_{2}-2 l, 1\right)
$$

see $[16,24,27,30]$. Similarly, the third flow (from (II) to (III)) has now the same structure as the non-perturbative analogue of (2.23), see [28, 29, 34, 35].

Remarkably, however, the form of the actual fixed point (IV) does not change for large values of $a_{2}$, and we find

$$
\left(a_{1}, a_{2}\right)_{m} \longrightarrow\left(a_{2}-1, a_{1}\right)_{m-1} \quad \text { for } \quad 1 \ll a_{2}<m-1,
$$

which is the same formula as (4.13). This suggests that the result for the endpoint- the fixed point (IV) — of the actual flow can be interpolated to intermediate values of $a_{2}$; a rather non-trivial test for this conjecture will be presented in section 4.4. Thus we are led to the final answer for the bulk-boundary flow

$$
\left(a_{1}, a_{2}\right)_{m} \longrightarrow\left\{\begin{array}{lll|}
\left(1, a_{1}\right)_{m-1} & \text { if } & a_{2}=1 \\
\left(a_{2}-1, a_{1}\right)_{m-1} & \text { if } & 1<a_{2}<m \\
\left(1, m-a_{1}\right)_{m-1} & \text { if } & a_{2}=m
\end{array}\right.
$$

under the $\phi_{(1,3)}$ bulk perturbation. We should mention that (4.20) reproduces in particular the conjectured flow of $[16,27]$

$$
(1, a)_{m} \longrightarrow(a-1,1)_{m-1}
$$

for $1<a<m$.

Finally, let us comment on the behaviour of the flows under the $\mathbb{Z}_{2}$ symmetry of the minimal models (see (4.6)). The bulk field $\phi_{(1,3)}$ is invariant under this symmetry (see (4.5)), and thus we expect the flows and fixed points to respect the symmetry ${ }^{9}$. In fact, it is not

9 One can explicitly check that the coupled RG equations remain invariant under the symmetry. There is a slight subtlety here, namely that the normalization of the boundary fields in [33] - this has an impact on the OPE coefficients that enter this calculation, see appendix A-depends on $m$. Once this has been taken into account, we find the same structures for the case of small $a_{1}$ and $a_{2}$ and for the $\mathbb{Z}_{2}$-image of this case. 
difficult to see that under the map $\left(a_{1}, a_{2}\right)_{m} \mapsto\left(a_{1}, m+1-a_{2}\right)_{m}$, the two sequences (4.15) and (4.17) are mapped into one another, and that the results (4.13) and (4.19) on the actual fixed point (IV) are left invariant.

\subsection{Consistency constraints}

Finally, there are a number of consistency constraints that we can check. They are all a variant of the following observation ${ }^{10}$. The flows from our initial boundary condition to the fixed point (IV) come in a one-parameter family labelled by a parameter $\chi$ that measures the initial strength of the boundary perturbation by $\psi_{(1,3)}$ relative to the bulk perturbation. (More precisely, $\chi$ is proportional to the initial value of the ratio $\mu / \sqrt{|\lambda|}$ when the perturbation is turned on, see (C.9) in appendix C.)

For large negative values of $\chi$, the flow first follows closely the pure boundary flow to (I), then the bulk flow down to (II) and finally the pure boundary flow from (II) to (IV) (see figure 1). In the limit $\chi \rightarrow-\infty$, the flow completely decomposes into three separate flows. If our analysis is correct, the separate flows should involve the same fixed points that we identified in our analysis. The fixed point (I) that is reached by the pure boundary perturbation is described by a superposition of boundary conditions $(b, 1)$. The subsequent bulk flow is thus given by (2.25). Combining these two flows, i.e. (2.19) and (2.25), then leads indeed to (2.21) with the same configuration at (II).

Similarly we can consider the limit $\chi \rightarrow+\infty$. Then we first follow a pure boundary flow that is the non-perturbative counterpart of the perturbative flow to (I), and we reach a fixed point $\left(\mathrm{I}^{\prime}\right)$ that corresponds to the superposition (4.18). For the subsequent bulk flow we can again use (2.25) and we arrive at a superposition ( $\left.\mathrm{II}^{\prime}\right)$ of boundary conditions of the form $\left(1, b_{i}\right)$. From there we can follow a pure boundary flow. Although we do not know $a$ priori which boundary $(1,3)$-fields are turned on, we know that at least there is a perturbative boundary flow (see (2.24)) involving in general also boundary condition changing fields that leads to the fixed point (IV) [28], see figure 2. To ensure that this is really the flow that takes place, one can again consider the case of $a_{1}=1$ where there is only one perturbative boundary $(1,3)$-flow from $\left(\mathrm{II}^{\prime}\right)$, and so it is easily identified. To get the flow for the general case, one then again uses the fusion of a defect (4.8).

This last boundary flow in the $c_{m-1}$ theory is actually the perturbative flow from (II') to (III') for another initial boundary condition $\mathbf{a}^{\prime}=\left(a_{1}, a_{2}-1\right)$. We can therefore combine the flow graphs for all boundary conditions $\left(a_{1}, \cdot\right)$ into a chain of flows; figure 2 shows a generic segment of the chain.

At the end of the chain, the situation degenerates: the boundary condition $\left(a_{1}, 2\right)_{m}$ flows to $\left(1, a_{1}\right)$, so the fixed point (IV) coincides with the fixed point $\left(\mathrm{II}^{\prime}\right)$ that is reached from the pure bulk flow from $\left(\mathrm{I}^{\prime}\right)$, see figure 3 .

The chains that we get for different values of $a_{1}$ all look similar. Indeed, the chain for general $a_{1}$ can be obtained starting from the chain with $a_{1}=1$ by fusion with a topological defect. The topological defect to consider is the one that corresponds to $\mathcal{D}\left(a_{1}, 1\right)_{m}$ in the $m$ th model. Along the upper sequence (i.e. in the $m$ th theory), this topological defect indeed maps the upper part of the $a_{1}=1$ chain to the upper part of the chain for $a_{1}$. As we have argued before, the defect $\mathcal{D}\left(a_{1}, 1\right)_{m}$ flows under the bulk flow to $\mathcal{D}\left(1, a_{1}\right)_{m-1}$, and it is therefore this topological defect that acts on the lower sequence. This then produces the lower sequence of the diagram for $a_{1}$.

${ }^{10}$ We thank Patrick Dorey for drawing our attention to this point. 


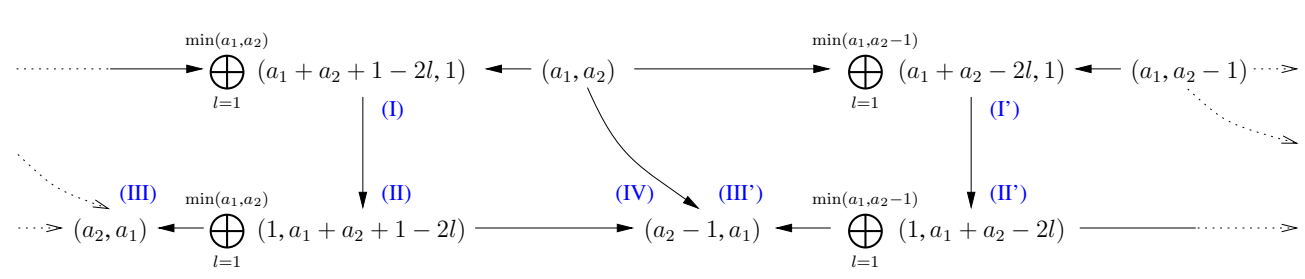

Figure 2. A segment of the chain of flows for small values of $a_{1}, a_{2}$. In the upper line we have boundary conditions of the $m$ th and in the lower line of the $(m-1)$ th minimal model.

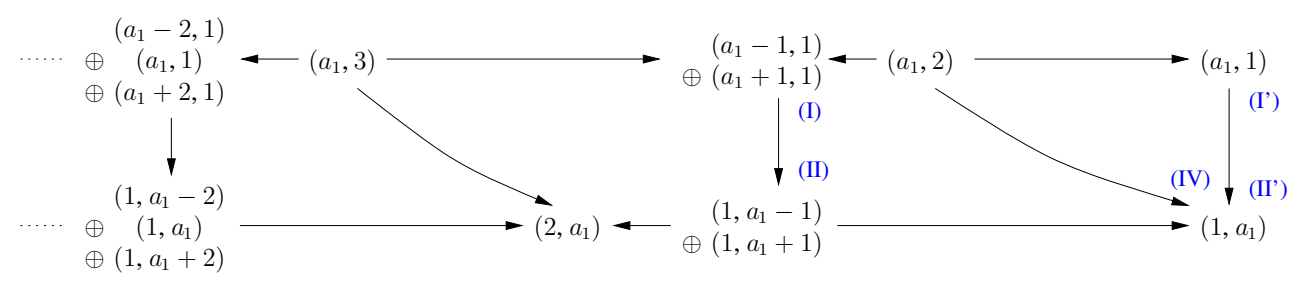

Figure 3. The right end of the chain of flows. Starting from the boundary condition $\left(a_{1}, 2\right)_{m}$, in one direction the flow decomposes into only two separate flows $\left(\rightarrow\left(a_{1}, 1\right)_{m} \rightarrow\left(1, a_{1}\right)_{m-1}\right)$ instead of three separate flows in the generic case.

\subsection{A perturbative analysis in the middle of the chain of flows}

When we extrapolate our chain from the right to larger values of $a_{2}$, the perturbative boundary flows become longer, and the non-perturbative flows become shorter. In the middle of the chain, they are of the same length, more precisely, for the boundary condition $\left(a_{1}, \frac{m+1}{2}\right)_{m}(m$ odd); the two boundary flows triggered by $\psi_{(1,3)}$ are mapped to each other by the $\mathbb{Z}_{2}$ symmetry and are thus equally long (see figure 4 ). The diagram suggests that for this boundary condition the pure bulk perturbation does not switch on the boundary field $\psi_{(1,3)}$. Indeed one finds that the bulk boundary coefficient $B_{(1,3)}^{(1,3)}=0$, so the fixed point is in this case given by $\lambda=\lambda_{*}$ and $\mu=0$. We can then compute the perturbed $g$-function, which to leading order only gets a contribution from the bulk one-point function. We find

$$
\log \frac{g_{\lambda_{*}, 0}^{(m)}\left(a_{1}, \frac{m+1}{2}\right)}{g^{(m)}\left(a_{1}, \frac{m+1}{2}\right)}=\frac{1}{4 m} \frac{2-3 \xi}{\xi}+\mathcal{O}\left(\xi, m^{-2}\right) .
$$

With our prescription for the cutoff $\xi$ (see (3.31)), we obtain the expected result

$$
\log \frac{g^{(m-1)}\left(\frac{m-1}{2}, a_{1}\right)}{g^{(m)}\left(a_{1}, \frac{m+1}{2}\right)}=-\frac{1}{2 m}+\mathcal{O}\left(m^{-2}\right) .
$$

This check gives support to the idea that our results can be extrapolated to any value of $a_{2}$. We can go even further and look at boundary conditions close to $\left(a_{1}, \frac{m+1}{2}\right)_{m}$. Figure 4 suggests that here the bulk boundary flow to the true fixed point (IV) is perturbative whereas the pure boundary flows are nonperturbative. To check this explicitly, let us consider the RG equations for the boundary coupling $\mu$ of the $\psi_{(1,3)}$ field for a boundary condition of the form $\mathbf{a}=\left(a_{1}, \frac{m+1}{2}-a_{2}\right)$,

$$
\begin{aligned}
\dot{\mu} & =(1-h) \mu+\frac{1}{2} B \lambda+E \lambda \mu+\cdots \\
& =\frac{2}{m} \mu-\pi a_{2} \lambda+4 \pi \lambda \mu+\cdots,
\end{aligned}
$$




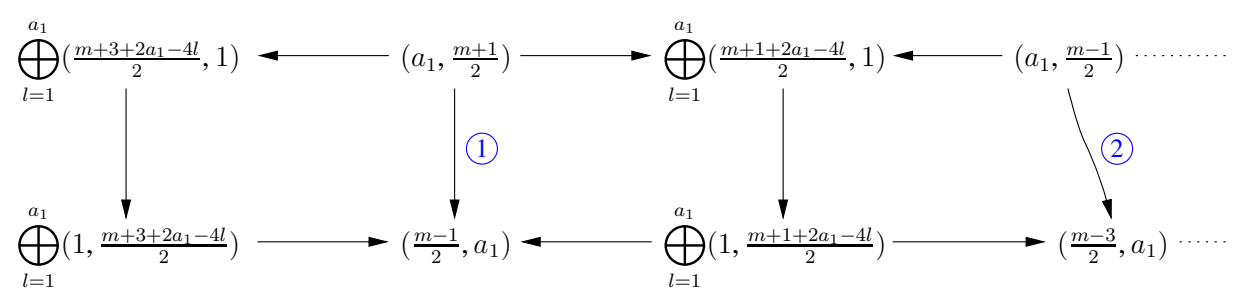

Figure 4. The middle of the chain of flows for $m$ odd. The fixed point starting from the exact middle $\left(a_{1}, \frac{m+1}{2}\right)_{m}$ is reached for $\lambda=\lambda_{*}, \mu=0$ (flow 1 in the figure). When we go slightly away from the middle (flow 2 ), the flow can still be treated perturbatively.

where $a_{1}$ and $a_{2}$ are now small. Here we have used the expressions for the coupling constants from appendix A.2. Note that we neglected the term proportional to $\mu^{2}$, because its coefficient $D_{(1,3)(1,3)}$ is suppressed by $m^{-3}$. For the calculation of $E$ we need to determine the correlator of the bulk field and two boundary fields to leading order in $1 / \mathrm{m}$, which now takes the form

$\frac{1}{\langle\mathbb{1}\rangle D_{(1,3)(1,3)}^{(1,1)}}\left\langle\phi_{(1,3)}(z, \bar{z}) \psi_{(1,3)}(0) \psi_{(1,3)}(\infty)\right\rangle=\frac{-\frac{3}{2}(z-\bar{z})^{4}+\frac{1}{2}(z+\bar{z})^{4}}{|z|^{4}(z-\bar{z})^{2}}+\mathcal{O}\left(m^{-1}\right)$.

In particular, this correlator has the correct asymptotics in the various channels: for the $(1,1)$ and $(1,5)$ channels, the relevant coefficients are now

$$
B_{(1,3)}^{(1,1)} D_{(1,1)(1,3)}^{(1,3)}=-1+\mathcal{O}\left(\frac{1}{m^{2}}\right), \quad B_{(1,3)}^{(1,5)} D_{(1,5)(1,3)}^{(1,3)}=\frac{4}{3}+\mathcal{O}\left(\frac{1}{m}\right),
$$

whereas the coefficient in the $(1,3)$ channel still vanishes to leading order

$$
B_{(1,3)}^{(1,3)} D_{(1,3)(1,3)}^{(1,3)}=\mathcal{O}\left(m^{-3}\right) .
$$

After integration as in (2.10), this leads to $E=4 \pi$. This is the expected value since at the fixed point $\lambda=\lambda_{*}=-\frac{1}{\pi m}$ the conformal dimension of the field coupling to $\mu$ should be $h_{(3,1)}=1+\frac{2}{m}+\cdots$.

For $\lambda=\lambda_{*}$, the RG equation (4.24) has a unique fixed point at $\mu_{*}=\frac{a_{2}}{2}$. This solution describes a perturbative fixed point. In fact the order 1 value of $\mu_{*}$ is only an artefact of our normalization since $D_{(1,3)(1,3)}$ is of order $m^{-2}$, and thus in the 'natural normalization' we should rescale the field $\psi$ by a factor of $m$, which would replace $\mu \mapsto \frac{\mu}{m}$. Note that after this rescaling, the boundary coupling constant $D_{(1,3)(1,3)}^{(1,3)}$ is only of order $m^{-2}$, and hence can still be ignored in the RG equation. It is also clear from the flow diagram (see figure 5) that this fixed point is actually reached by a generic flow.

Using the same techniques as above in section 3, we can now determine the change in the $g$-factor:

$$
\begin{aligned}
\log g_{\lambda_{*}, \mu_{*}}^{(m)}(\mathbf{a}) & =\log g^{(m)}(\mathbf{a})-\frac{16 \pi^{2}}{m^{3}} \mu_{*}^{2}-\frac{2 \pi^{3} a_{2}^{2}}{m^{2}} \lambda_{*}\left(1-\frac{8}{a_{2}} \mu_{*}+\frac{16}{a_{2}^{2}} \mu_{*}^{2}\right)+\cdots \\
& =\log g^{(m)}(\mathbf{a})-\frac{2 \pi^{2} a_{2}^{2}}{m^{3}}+\cdots,
\end{aligned}
$$

where the dots denote contributions that are either of higher order in $1 / \mathrm{m}$ or do not depend on the boundary labels. To remove the terms that are independent of the boundary labels, we again look at relative $g$-functions with respect to some reference boundary condition, which we choose here to be $\left(1, \frac{m+1}{2}\right)_{m}$ (note that we have to use a boundary condition which is in the regime where the perturbative analysis applies). The relative $g$-function in the $m$ th model is

$$
\frac{g^{(m}(\mathbf{a})}{g^{(m)}\left(1, \frac{m+1}{2}\right)}=\frac{\sin \frac{\pi a_{1}}{m}}{\sin \frac{\pi}{m}}\left(1-\frac{\pi^{2} a_{2}^{2}}{2 m^{2}}+\frac{\pi^{2} a_{2}^{2}}{m^{3}}+\mathcal{O}\left(m^{-4}\right)\right) \text {. }
$$




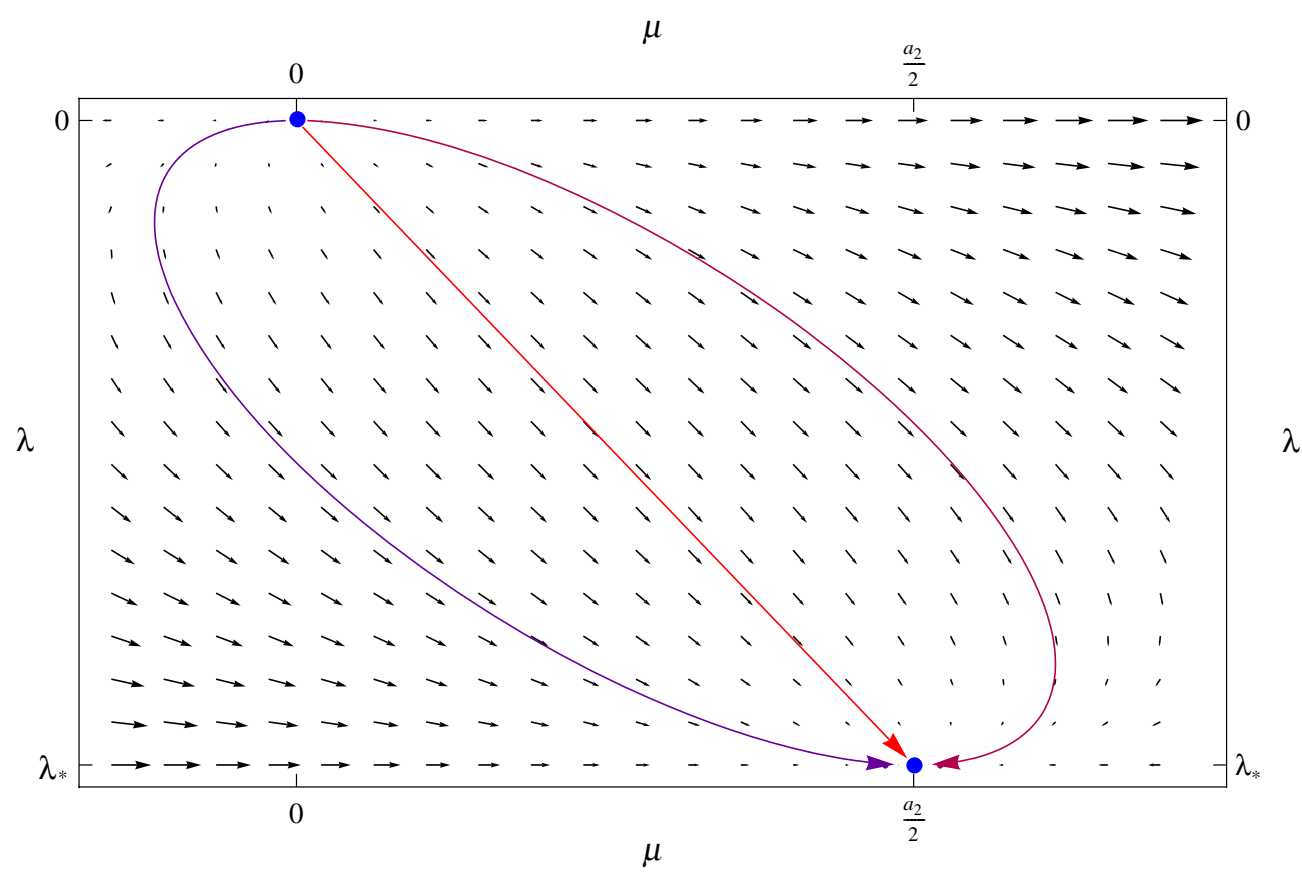

Figure 5. The flow diagram for (4.24) for $a_{2}=1$ and $m=101$. The vectors $(\dot{\mu}, \dot{\lambda})$ have been magnified by a factor 2.5. A generic flow (described by solution (C.12) of (4.24)) reaches the perturbative fixed point.

After the perturbation by $\phi_{(1,3)}$ the relative $g$-function becomes

$$
\begin{aligned}
\log \frac{g_{\lambda_{*}, \mu_{*}}^{(m)}(\mathbf{a})}{g_{\lambda_{*}, 0}^{(m)}\left(1, \frac{m+1}{2}\right)} & =\log \frac{\sin \frac{\pi a_{1}}{m}}{\sin \frac{\pi}{m}}-\frac{\pi^{2} a_{2}^{2}}{2 m^{2}}-\frac{\pi^{2} a_{2}^{2}}{m^{3}}+\mathcal{O}\left(m^{-4}\right) \\
& =\log \frac{g^{(m-1)}\left(\frac{m-1}{2}-a_{2}, a_{1}\right)}{g^{(m-1)}\left(\frac{m-1}{2}, 1\right)}+\mathcal{O}\left(m^{-4}\right) .
\end{aligned}
$$

This perturbative calculation thus predicts that the endpoint of the actual flow is

$$
\left(a_{1}, \frac{m+1}{2}-a_{2}\right)_{m} \stackrel{\text { (IV) }}{\longrightarrow}\left(\frac{m-1}{2}-a_{2}, a_{1}\right)_{m-1} .
$$

It is remarkable that in this case, the actual endpoint of the flow (i.e. the fixed point (IV)) can be directly computed perturbatively. The result (4.32) is in beautiful agreement with our general answer (4.20), and thus gives strong support for the claim that (4.20) is also true for intermediate values of $a_{2}$.

\section{Comparison with numerical results}

Finally let us analyse how our perturbative calculation compares with numerical calculations that have been performed before. In particular, in $[25,26]$, the flow of the tricritical Ising $(m=4)$ to the Ising model $(m=3)$ was considered in the presence of boundaries. They considered a cylinder diagram with two boundaries for which they imposed the boundary conditions $(r, 1)$ and $(1, s)$, respectively. According to the fusion rules, the relative open string 
spectrum between the two boundary conditions transforms then in the $(r, s)$ representation, and they found that under the $\phi_{(1,3)}$ flow, the open string character $\chi_{(r, s)}$ flowed as ${ }^{11}$

$$
\begin{array}{lll}
m=4 & & m=3 \\
\chi_{(1,1)} & \longrightarrow & \chi_{(1,1)} \\
\chi_{(2,1)} & \longrightarrow & \chi_{(1,2)} \\
\chi_{(3,1)} & \longrightarrow & \chi_{(1,3)} \\
\chi_{(1,2)} & \longrightarrow & \chi_{(1,1)} \\
\chi_{(1,3)} & \longrightarrow & \chi_{(2,1)} \\
\chi_{(2,2)} & \longrightarrow & \chi_{(1,2)} .
\end{array}
$$

In particular, the first flow implies that the $(1,1)$ boundary condition of the $m=4$ theory flows to the $(1,1)$ boundary condition of the $m=3$ theory. In order to compare with our calculations (where we consider a single boundary condition), we can take either $r=1$ or $s=1$ since then one of the two boundary conditions does not flow. In this way we deduce from (5.1) that the boundary conditions flow as

$$
\begin{aligned}
& (1,1)_{4} \longrightarrow(1,1)_{3} \\
& (2,1)_{4} \longrightarrow(1,2)_{3} \\
& (3,1)_{4} \longrightarrow(1,3)_{3} \\
& (1,2)_{4} \longrightarrow(1,1)_{3} \\
& (1,3)_{4} \longrightarrow(2,1)_{3} \cong(1,3)_{3}
\end{aligned}
$$

Unfortunately, we cannot determine the flow of the $(2,2)$ boundary condition in this manner, since none of the cylinder diagrams considered in $[25,26]$ involves this boundary condition. However, one may suspect that the behaviour of the cylinder diagram between $(1,1)$ and $(2,2)$ should be the same as that between $(1,2)$ and $(2,1)$. Following this line of argument would lead to the prediction $(2,2)_{4} \rightarrow(1,2)_{3}$, which is indeed in agreement with our general rule (4.20).

The flows (5.2) agree perfectly with our general rule (4.20). A schematic view of the flows in the space of couplings $\lambda, \mu$ is as follows:

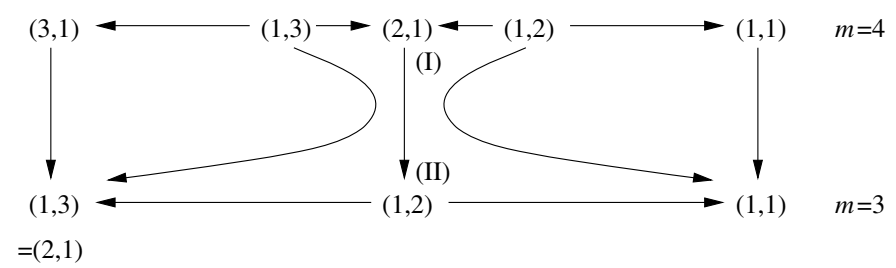

The three vertical flows (no boundary fields are switched on) are the first three flows in (5.2). The horizontal flows are the well-known boundary flows in the $m=4$ and $m=3$ model, respectively, which are driven by the $\psi_{(1,3)}$ boundary field. The short horizontal arrows in the $m=4$ model indicate that these flows become the perturbative $\psi_{(1,3)}$ flows when $m$ is sent to large values ${ }^{12}$. Finally, the flows indicated by the curved arrows agree precisely with the last two flows of (5.2).

This final check of our general prediction (4.20) at this low value of $m$ makes us confident that our results hold for the whole (A-type) series of unitary minimal models.

\footnotetext{
${ }^{11}$ Note that there is a typographical error in table 1 of [26]. As explained in the accompanying text of [26], the flow of the characters $\chi_{(1,2)}$ and $\chi_{(1,3)}$ in the $m=4$ model is rather as indicated in (5.1). We thank Leung Chim for pointing this out to us.

${ }^{12}$ The flow $(1,2)_{4} \rightarrow(2,1)_{4}$ becomes $(1,2)_{m} \rightarrow(2,1)_{m}$ for large $m$, while the flow $(1,3)_{4} \rightarrow(2,1)_{4}$ becomes $(1, m-1)_{m} \rightarrow(m-2,1)_{m}$.
} 


\section{Acknowledgments}

We are indebted to Patrick Dorey, Anatoly Konechny and Ingo Runkel for detailed explanations and discussions. We also thank Albion Lawrence and Volker Schomerus for initial collaboration on this project, and Andreas Recknagel for useful comments. The work of MRG and CSC is supported by the Swiss National Science Foundation. MRG thanks the GGI in Florence, the Chinese Academy of Sciences in Beijing and the IPMU in Tokyo for hospitality during the final stages of this work.

\section{Appendix A. OPE coefficients}

For the charge conjugation minimal model (the A-series), the various OPE coefficients have been determined in [33] in terms of the $F$ matrices. For large $m$ the bulk coupling constant $C_{(1,3)(1,3)}{ }_{(1,3)}$ has the following large $m$ behaviour:

$$
C_{(1,3)(1,3)}^{(1,3)}=4-\frac{12}{m}-\frac{8}{3}\left(3+\pi^{2}\right) \frac{1}{m^{2}}+\mathcal{O}\left(m^{-3}\right) .
$$

We also need the large $m$ expansions of the boundary and bulk-boundary OPE coefficients.

\section{A.1. The expansion for small $a_{1}, a_{2}$}

For a boundary condition $a=\left(a_{1}, a_{2}\right)$ for which $a_{1}, a_{2} \ll m$, we have the following asymptotic expansions of the OPE coefficients:

$$
\begin{aligned}
& D_{(1,1)(1,3)}^{(1,3)}= 1, \\
& D_{(1,3)(1,3)}^{(1,1)}= \begin{cases}\frac{2\left(a_{2}-1\right)}{\left(a_{1}-a_{2}\right)^{2}\left(a_{2}+1\right)}+\mathcal{O}\left(\frac{1}{m}\right) & \left(a_{2}>a_{1}\right) \\
\frac{2 m^{2}}{\left(a_{2}^{2}-1\right)}-\frac{6 m}{a_{2}^{2}-1}+\frac{4}{a_{2}^{2}-1}+\mathcal{O}\left(\frac{1}{m}\right) & \left(a_{2}=a_{1}\right) \\
\frac{2\left(a_{2}+1\right)}{\left(a_{1}-a_{2}\right)^{2}\left(a_{2}-1\right)}+\mathcal{O}\left(\frac{1}{m}\right) & \left(a_{2}<a_{1}\right),\end{cases}
\end{aligned}
$$

$D_{(1,3)(1,3)}^{(1,3)}=\left\{\begin{array}{lc}-\frac{4}{\left(a_{1}-a_{2}\right)\left(a_{2}+1\right)}+\mathcal{O}\left(\frac{1}{m}\right) & \left(a_{2}>a_{1}\right) \\ -\frac{4 m}{\left(a_{2}^{2}-1\right)}+\mathcal{O}(1) & \left(a_{2}=a_{1}\right) \\ -\frac{4}{\left(a_{1}-a_{2}\right)\left(a_{2}-1\right)}+\mathcal{O}\left(\frac{1}{m}\right) & \left(a_{2}<a_{1}\right),\end{array}\right.$

$$
D_{(1,5)(1,3)}^{(1,3)}= \begin{cases}-\frac{24\left(a_{2}-2\right)}{\left(a_{2}+1\right)\left(a_{1}-a_{2}\right)^{2}\left(\left(a_{1}-a_{2}\right)^{2}-1\right)}+\mathcal{O}\left(\frac{1}{m}\right) & \left(a_{2} \geqslant a_{1}+2\right) \\ \frac{12 m}{a_{1}+2}+\frac{2\left(15 a_{1}-34\right)}{a_{1}+2}+\mathcal{O}\left(\frac{1}{m}\right) & \left(a_{2}=a_{1}+1\right) \\ \frac{24 m^{2}}{a_{1}^{2}-1}-\frac{172 m}{a_{1}^{1}-1}+\frac{4\left(6 a_{1}^{2}+77+12 \pi^{2}\right)}{a_{1}^{2}-1}+\mathcal{O}\left(\frac{1}{m}\right) & \left(a_{2}=a_{1}\right) \\ -\frac{12 m}{a_{1}-2}+\frac{2\left(15 a_{1}+34\right)}{a_{1}-2}+\mathcal{O}\left(\frac{1}{m}\right) & \left(a_{2}=a_{1}-1\right) \\ -\frac{24\left(a_{2}+2\right)}{\left(a_{2}-1\right)\left(a_{1}-a_{2}\right)^{2}\left(\left(a_{1}-a_{2}\right)^{2}-1\right)}+\mathcal{O}\left(\frac{1}{m}\right) & \left(a_{2} \leqslant a_{1}-2\right),\end{cases}
$$




$$
D_{(1,5)(1,5)}^{(1,1)}= \begin{cases}\frac{864\left(a_{2}-1\right)\left(a_{2}-2\right)}{\left(a_{2}+1\right)\left(a_{2}+2\right)\left(a_{1}-a_{2}\right)^{4}\left(\left(a_{1}-a_{2}\right)^{2}-1\right)^{2}}+\mathcal{O}\left(\frac{1}{m}\right) & \left(a_{2} \geqslant a_{1}+2\right) \\ \frac{216 a_{1} m^{2}}{\left(a_{1}+3\right)\left(a_{1}+2\right)\left(a_{1}-1\right)}+\mathcal{O}(m) & \left(a_{2}=a_{1}+1\right) \\ \frac{864 m^{4}}{\left(a_{1}+2\right)\left(a_{1}+1\right)\left(a_{1}-1\right)\left(a_{1}-2\right)}+\mathcal{O}\left(m^{3}\right) & \left(a_{2}=a_{1}\right) \\ \frac{216 a_{1} m^{2}}{\left(a_{1}+1\right)\left(a_{1}-2\right)\left(a_{1}-3\right)}+\mathcal{O}(m) & \left(a_{2}=a_{1}-1\right) \\ \frac{864\left(a_{2}+1\right)\left(a_{2}+2\right)}{\left(a_{2}-1\right)\left(a_{2}-2\right)\left(a_{1}-a_{2}\right)^{4}\left(\left(a_{1}-a_{2}\right)^{2}-1\right)^{2}}+\mathcal{O}\left(\frac{1}{m}\right) & \left(a_{2} \leqslant a_{1}-2\right),\end{cases}
$$

$$
D_{(1,5)(1,5)}^{(1,3)}= \begin{cases}-\frac{5184\left(a_{2}-2\right)}{\left(\left(a_{1}-a_{2}\right)^{2}-1\right)^{2}\left(a_{1}-a_{2}\right)^{3}\left(a_{2}+1\right)\left(a_{2}+2\right)}+\mathcal{O}\left(\frac{1}{m}\right) & \left(a_{2} \geqslant a_{1}+2\right) \\ \frac{1296 m^{2}}{\left(a_{1}+3\right)\left(a_{1}+2\right)\left(a_{1}-1\right)}+\mathcal{O}(m) & \left(a_{2}=a_{1}+1\right) \\ -\frac{5184 m^{3}}{\left(a_{1}+2\right)\left(a_{1}+1\right)\left(a_{1}-1\right)\left(a_{1}-2\right)}+\mathcal{O}\left(m^{2}\right) & \left(a_{2}=a_{1}\right) \\ -\frac{1296 m^{2}}{\left(a_{1}-3\right)\left(a_{1}-2\right)\left(a_{1}+1\right)}+\mathcal{O}(m) & \left(a_{2}=a_{1}-1\right) \\ -\frac{5184\left(a_{2}+2\right)}{\left(\left(a_{1}-a_{2}\right)^{2}-1\right)^{2}\left(a_{1}-a_{2}\right)^{3}\left(a_{2}-1\right)\left(a_{2}-2\right)}+\mathcal{O}\left(\frac{1}{m}\right) & \left(a_{2} \leqslant a_{1}-2\right),\end{cases}
$$

$$
B_{(1,3)}^{(1,1)}=3-\frac{4 \pi^{2}}{m^{2}} a_{2}^{2}+\mathcal{O}\left(\frac{1}{m^{3}}\right),
$$

$$
\begin{aligned}
& B_{(1,3)}^{(1,3)}= \begin{cases}\frac{4 \pi}{m}\left(a_{1}-a_{2}\right)\left(a_{2}+1\right)+\mathcal{O}\left(\frac{1}{m^{2}}\right) & \left(a_{2}>a_{1}\right) \\
\frac{4 \pi}{m^{2}}\left(a_{2}^{2}-1\right)+\mathcal{O}\left(\frac{1}{m^{3}}\right) & \left(a_{2}=a_{1}\right) \\
\frac{4 \pi}{m}\left(a_{1}-a_{2}\right)\left(a_{2}-1\right)+\mathcal{O}\left(\frac{1}{m^{2}}\right) & \left(a_{2}<a_{1}\right),\end{cases} \\
& B_{(1,3)}^{(1,5)}= \begin{cases}-\frac{\pi^{2}}{18 m^{2}}\left(a_{2}+1\right)\left(a_{2}+2\right)\left(a_{1}-a_{2}\right)^{2}\left(\left(a_{1}-a_{2}\right)^{2}-1\right)+\mathcal{O}\left(\frac{1}{m^{3}}\right) & \left(a_{2} \geqslant a_{1}+2\right) \\
\frac{\pi^{2}}{9 m^{3}}\left(a_{1}-1\right)\left(a_{1}+2\right)\left(a_{1}+3\right)+\mathcal{O}\left(\frac{1}{m^{4}}\right) & \left(a_{2}=a_{1}+1\right) \\
\frac{\pi^{2}}{18 m^{4}}\left(a_{1}^{2}-4\right)\left(a_{1}^{2}-1\right)+\mathcal{O}\left(\frac{1}{m^{5}}\right) & \left(a_{2}=a_{1}\right) \\
-\frac{\pi^{2}}{9 m^{3}}\left(a_{1}+1\right)\left(a_{1}-2\right)\left(a_{1}-3\right)+\mathcal{O}\left(\frac{1}{m^{4}}\right) & \left(a_{2} \leqslant a_{1}-2\right) . \\
-\frac{\pi^{2}}{18 m^{2}}\left(a_{2}-1\right)\left(a_{2}-2\right)\left(a_{1}-a_{2}\right)^{2}\left(\left(a_{1}-a_{2}\right)^{2}-1\right)+\mathcal{O}\left(\frac{1}{m^{3}}\right)\end{cases}
\end{aligned}
$$




\section{A.2. Asymptotic expansion for $a_{1}$ small and $a_{2}$ close to $\frac{m+1}{2}$}

For boundary conditions of the form $\left(a_{1}, \frac{m+1}{2}-a_{2}\right)_{m}$ where $m$ is odd and $a_{1}, a_{2} \ll m$, we have on the other hand

$$
\begin{aligned}
& D_{(1,3)(1,3)}^{(1,1)}=\frac{8}{m^{2}}+\mathcal{O}\left(\frac{1}{m^{3}}\right) \\
& D_{(1,3)(1,3)}^{(1,3)}=\frac{8 \pi^{2} a_{2}}{m^{3}}+\mathcal{O}\left(\frac{1}{m^{4}}\right) \\
& D_{(1,1)(1,3)}^{(1,3)}=1 \\
& D_{(1,5)(1,3)}^{(1,3)}=-\frac{384}{m^{4}}+\mathcal{O}\left(\frac{\log m}{m^{5}}\right) \\
& D_{(1,5)(1,5)}^{(1,1)}=\frac{9 \times 2^{13}}{m^{8}}+\mathcal{O}\left(\frac{\log m}{m^{9}}\right) \\
& D_{(1,5)(1,5)}^{(1,3)}=2^{13} \times 3^{4} \pi^{2} a_{2} \frac{1}{m^{9}}+\mathcal{O}\left(\frac{\log m}{m^{10}}\right) \\
& B_{(1,3)}^{(1,1)}=-1+\frac{4 \pi^{2} a_{2}^{2}}{m^{2}}+\mathcal{O}\left(\frac{1}{m^{3}}\right) \\
& B_{(1,3)}^{(1,3)}=-2 \pi a_{2}+\mathcal{O}\left(\frac{1}{m}\right) \\
& B_{(1,3)}^{(1,5)}=-\frac{m^{4}}{288}+\mathcal{O}\left(\log (m) m^{3}\right)
\end{aligned}
$$

\section{Appendix B. Correlation functions and integrals}

\section{B.1. Disc and upper half-plane}

The upper half-plane is defined by $\mathbb{H}^{+}=\{z \in \mathbb{C} \mid \operatorname{Im} z \geqslant 0\}$, while the disc is defined by $\mathbb{D}=\{w \in \mathbb{C}|| w \mid \leqslant 1\}$. On the disc, we often use the polar coordinates $w=r \mathrm{e}^{\mathrm{i} \theta}$, where $0 \leqslant r \leqslant 1$ and $-\pi<\theta \leqslant \pi$. The transition function from $\mathbb{H}^{+}$to $\mathbb{D}$ is given by

$$
z(w)=\mathrm{i} \frac{1-w}{1+w} .
$$

Primary fields of the CFT are denoted $\phi$ in the bulk, with conformal dimension $\Delta=2 h$, and $\psi$ on the boundary, with conformal dimension $h$. The identity field is denoted by $\mathbb{1}$, both in the bulk and on the boundary. The basic correlators on the upper half-plane are

$$
\begin{aligned}
& \langle\psi(x)\rangle_{\mathbb{H}^{+}}=\delta_{\psi, \mathbb{1}}\langle\mathbb{1}\rangle_{\mathbb{H}^{+}}, \\
& \left\langle\psi\left(x_{1}\right) \psi\left(x_{2}\right)\right\rangle_{\mathbb{H}^{+}}=D_{\psi \psi}{ }^{11}\langle\mathbb{1}\rangle_{\mathbb{H}^{+}}\left(x_{1}-x_{2}\right)^{-2 h} \quad\left(x_{1}>x_{2}\right), \\
& \langle\phi(x+\mathrm{i} y, x-\mathrm{i} y)\rangle_{\mathbb{H}^{+}}=B_{\phi}{ }^{\mathbb{1}}\langle\mathbb{1}\rangle_{\mathbb{H}^{+}}(2 y)^{-\Delta} \quad(y>0), \\
& \left\langle\phi\left(x_{1}+\mathrm{i} y_{1}, x_{1}-\mathrm{i} y_{1}\right) \psi\left(x_{2}\right)\right\rangle_{\mathbb{H}^{+}}=B_{\phi}{ }^{\psi} D_{\psi \psi}{ }^{11}\langle\mathbb{1}\rangle_{\mathbb{H}^{+}}\left(2 y_{1}\right)^{-\Delta+h}\left(\left(x_{1}-x_{2}\right)^{2}+y_{1}^{2}\right)^{-h} \quad\left(y_{1}>0\right), \\
& \left\langle\psi_{1}\left(x_{1}\right) \psi_{2}\left(x_{2}\right) \psi_{3}\left(x_{3}\right)\right\rangle_{\mathbb{H}^{+}}=D_{\psi_{2} \psi_{3}}{ }^{\psi_{1}} D_{\psi_{1} \psi_{1}}{ }^{1}\langle\mathbb{1}\rangle_{\mathbb{H}^{+}}\left(x_{1}-x_{2}\right)^{h_{3}-h_{2}-h_{1}} \\
& \quad \times\left(x_{2}-x_{3}\right)^{h_{1}-h_{2}-h_{3}}\left(x_{1}-x_{3}\right)^{h_{2}-h_{1}-h_{3}} \quad\left(x_{1}>x_{2}>x_{3}\right) .
\end{aligned}
$$


On the disc, these are

$$
\begin{aligned}
& \left\langle\psi\left(r \mathrm{e}^{\mathrm{i} \theta}\right)\right\rangle_{\mathbb{D}}=\delta_{\psi, \mathbb{1}}\langle\mathbb{1}\rangle_{\mathbb{D}}, \\
& \left\langle\psi\left(\mathrm{e}^{\mathrm{i} \theta_{1}}\right) \psi\left(\mathrm{e}^{\mathrm{i} \theta_{2}}\right)\right\rangle_{\mathbb{D}}=D_{\psi \psi}{ }^{\mathbb{1}}\langle\mathbb{1}\rangle_{\mathbb{D}}\left|2 \sin \frac{\theta_{1}-\theta_{2}}{2}\right|^{-2 h}, \\
& \left\langle\phi\left(r \mathrm{e}^{\mathrm{i} \theta}, r \mathrm{e}^{-\mathrm{i} \theta}\right)\right\rangle_{\mathbb{D}}=B_{\phi}{ }^{\mathbb{1}}\langle\mathbb{1}\rangle_{\mathbb{D}}\left(1-r^{2}\right)^{-\Delta}, \\
& \left\langle\phi\left(r \mathrm{e}^{\mathrm{i} \theta_{1}}, r \mathrm{e}^{-\mathrm{i} \theta_{1}}\right) \psi\left(\mathrm{e}^{\mathrm{i} \theta_{2}}\right)\right\rangle_{\mathbb{D}}=B_{\phi}{ }^{\psi} D_{\psi \psi}{ }^{\mathbb{1}}\langle\mathbb{1}\rangle_{\mathbb{D}}\left(1-r^{2}\right)^{-\Delta}\left(\frac{1-r^{2}}{1-2 r \cos \left(\theta_{1}-\theta_{2}\right)+r^{2}}\right)^{h}, \\
& \left\langle\psi_{1}\left(\mathrm{e}^{\mathrm{i} \theta_{1}}\right) \psi_{2}\left(\mathrm{e}^{\mathrm{i} \theta_{2}}\right) \psi_{3}\left(\mathrm{e}^{\mathrm{i} \theta_{3}}\right)\right\rangle_{\mathbb{D}}=D_{\psi_{2} \psi_{3}}{ }^{\psi_{1}} D_{\psi_{1} \psi_{1}}{ }^{1}\langle\mathbb{1}\rangle_{\mathbb{D}}\left|2 \sin \frac{\theta_{1}-\theta_{2}}{2}\right|^{h_{3}-h_{2}-h_{1}} \\
& \times\left|2 \sin \frac{\theta_{2}-\theta_{3}}{2}\right|^{h_{1}-h_{2}-h_{3}}\left|2 \sin \frac{\theta_{1}-\theta_{3}}{2}\right|^{h_{2}-h_{1}-h_{3}} .
\end{aligned}
$$

In sections 2.1 and 3, we also need an expression for the correlator

$$
\langle\phi(z, \bar{z}) \psi(x) \psi(y)\rangle_{\mathbf{a}}
$$

in the limit $m \rightarrow \infty$. This is a chiral four-point function which can be computed from the differential equation associated with the singular vector of the $(1,3)$ module at level 3:

$$
\mathcal{N}=\left(L_{-3}-2 h^{-1} L_{-2} L_{-1}+\frac{(m+1)^{2}}{2 m(m-1)}\left(L_{-1}\right)^{3}\right) \phi .
$$

The general solution to the corresponding third-order differential equation in the cross-ratio $\eta$ can be obtained in the limit $m \rightarrow \infty$. One can fix the solution by the asymptotic behaviour when the bulk-field approaches the boundary, but as the dimensions of the fields in the asymptotic channels differ by integers in the limit $m \rightarrow \infty$, this is not the easiest method. A better approach is to solve the differential equation for finite $m$ in an expansion around $\eta=0$ where the channels can be clearly separated, and then match the two sets of solutions in the limit $m \rightarrow \infty$. Of the three conformal blocks associated with the channels $(1,1),(1,3)$ and $(1,5)$, only the one involving the identity has a nontrivial contribution for $m \rightarrow \infty$. On the upper half-plane, we find

$$
\langle\phi(z, \bar{z}) \psi(x) \psi(y)\rangle_{\mathbb{H}^{+}}=\frac{\eta_{1}^{4}-3\left(\eta_{2}^{4}+\eta_{3}^{4}\right)}{6\left(\eta_{1} \eta_{2} \eta_{3}\right)^{2}} B_{(1,3)}^{(1,1)} D_{(1,1)(1,3)}^{(1,3)} D_{(1,3)(1,3)}^{(1,1)}\langle\mathbb{1}\rangle_{\mathbb{H}^{+}}\left(1+\mathcal{O}\left(m^{-1}\right)\right)
$$

where

$\eta_{1}=(z-\bar{z})(x-y), \quad \eta_{2}=(z-x)(\bar{z}-y), \quad \eta_{3}=\eta_{2}-\eta_{1}=(z-y)(\bar{z}-x)$.

Multiplying with $y^{2}$, this yields (2.11) in the limit $x \rightarrow 0, y \rightarrow \infty$. To perform the integral that leads to (3.16), we need an expression for the correlator on the disc:

$\left\langle\phi(w, \bar{w}) \psi\left(\mathrm{e}^{\mathrm{i} \theta_{1}}\right) \psi\left(\mathrm{e}^{\mathrm{i} \theta_{2}}\right)\right\rangle_{\mathbb{D}}=\frac{\eta_{1}^{\prime 4}-3\left(\eta_{2}^{\prime 4}+\eta_{3}^{\prime 4}\right)}{6\left(\eta_{1}^{\prime} \eta_{2}^{\prime} \eta_{3}^{\prime}\right)^{2}} B_{(1,3)}^{(1,1)} D_{(1,1)(1,3)}^{(1,3)} D_{(1,3)(1,3)}^{(1,1)}\langle\mathbb{1}\rangle_{\mathbb{H}^{+}}\left(1+\mathcal{O}\left(m^{-1}\right)\right)$,

where

$$
\begin{aligned}
& \eta_{1}^{\prime}=\left(1-|w|^{2}\right)\left(\mathrm{e}^{\mathrm{i} \frac{\theta_{2}-\theta_{1}}{2}}-\mathrm{e}^{-\mathrm{i} \frac{\theta_{2}-\theta_{1}}{2}}\right), \quad \eta_{2}^{\prime}=\mathrm{e}^{\mathrm{i} \frac{\theta_{1}-\theta_{2}}{2}}\left(w \mathrm{e}^{-\mathrm{i} \theta_{1}}-1\right)\left(1-\bar{w} \mathrm{e}^{\mathrm{i} \theta_{2}}\right), \\
& \eta_{3}^{\prime}=\eta_{2}^{\prime}-\eta_{1}^{\prime}=\mathrm{e}^{\mathrm{i} \frac{\theta_{2}-\theta_{1}}{2}}\left(w \mathrm{e}^{-\mathrm{i} \theta_{2}}-1\right)\left(1-\bar{w} \mathrm{e}^{\mathrm{i} \theta_{1}}\right) .
\end{aligned}
$$


Integrating this expression with our cut-offs $\epsilon$ on the boundary and $\xi$ between bulk and boundary, we find

$$
\begin{aligned}
\frac{2 \pi}{3} B_{(1,3)}^{(1,1)} D_{(1,1)(1,3)} D_{(1,3)(1,3)}^{(1,1)}\langle\mathbb{1}\rangle_{\mathbb{D}}\left(1+\mathcal{O}\left(m^{-1}\right)\right) \\
\quad \times\left(\left(\frac{3 \pi(2-3 \xi)}{2 \xi}+\mathcal{O}(\xi)\right) \frac{1}{\epsilon}+\left(\frac{2 \pi^{2}(3 \xi-2)}{\xi}+\mathcal{O}(\xi)\right)\right. \\
\left.+\left(\frac{\pi}{\xi^{2}}-\frac{5 \pi}{4 \xi}+\frac{\pi}{8}+\mathcal{O}(\xi)\right) \epsilon+\mathcal{O}\left(\epsilon^{2}\right)\right) .
\end{aligned}
$$

The term proportional to $\epsilon^{-1}$ is subtracted when we compute the connected correlation function. The $\epsilon$-independent part, on the other hand, gives our result (3.16).

\section{B.2. Semi-infinite cylinder}

Instead of considering the theory on the disc, we now put it on a semi-infinite cylinder by the conformal map

$$
w=r \mathrm{e}^{\mathrm{i} \theta} \mapsto v=-\log w=-\log r-\mathrm{i} \theta=: v_{1}+\mathrm{i} v_{2} .
$$

The coordinate $v_{1}$ runs from 0 to $\infty$, whereas $v_{2}$ is periodic with period $2 \pi$. A bulk one-point function in these coordinates reads

$$
\langle\Phi(v, \bar{v})\rangle_{\mathrm{C}}=B_{\phi}{ }^{11}\langle\mathbb{1}\rangle\left(2 \sinh v_{1}\right)^{-\Delta}
$$

Let us consider the integral of a one-point function of a marginal bulk field over the semiinfinite cylinder. To regularize the integral we have to introduce a cutoff $\eta$ such that $v_{1}$ cannot come too close to the boundary. This leads to

$I_{C}=\int_{\eta}^{\infty} \mathrm{d} v_{1}\left(2 \sinh v_{1}\right)^{-2}=\frac{1}{4}(-1+\operatorname{coth} \eta)=\frac{1}{4}\left(\frac{1}{\eta}-1+\mathcal{O}(\eta)\right)$.

Comparing this with the disc integral $I_{3}$ in (3.11) that appeared in the computation of the one-point contribution to the $g$-factor, we see that we get coinciding results precisely when we relate the cutoffs $\xi$ and $\eta$ as in (3.31). We conclude that on the semi-infinite cylinder the natural cutoff $\eta$ is already the right one. One could have expected this because here bulk and boundary contributions should be disentangled as the boundary is not curved with respect to the bulk metric, in contrast to the disc ${ }^{13}$.

\section{Appendix C. Solutions of the RG equations}

The set of RG equations in (2.16),

$$
\begin{aligned}
& \dot{\lambda}=\frac{4}{m} \lambda+4 \pi \lambda^{2}, \\
& \dot{\mu}=\frac{2}{m} \mu+\frac{2 \pi \alpha}{m} \lambda-4 \pi \lambda \mu-\frac{4}{\alpha} \mu^{2},
\end{aligned}
$$

can be solved explicitly. First note that (C.1) has the general solution

$$
\lambda(t)=\lambda_{*} \frac{C \mathrm{e}^{\frac{4}{m} t}}{1+C \mathrm{e}^{\frac{4}{m} t}}, \quad \lambda_{*}=-\frac{1}{\pi m},
$$

${ }^{13}$ This observation and the preceding computation resulted from discussions with Anatoly Konechny. 
where $C$ is some constant. For the flows to connect $\lambda=0$ at $t \rightarrow-\infty$ and $\lambda=\lambda_{*}$ at $t \rightarrow+\infty$, we have to take $C>0$. In that case, $\lambda$ flows strictly monotonically, and we can parameterize

$$
\mu(t)=\pi \alpha f(\lambda(t)),
$$

with some function $f(\lambda)$. Differentiating (C.4) with respect to $t$ and plugging in (C.1) and (C.2), we obtain an ordinary first-order differential equation for $f(\lambda)$ :

$$
2 \lambda(1+\pi m \lambda) f^{\prime}(\lambda)=f(\lambda)+\lambda-2 \pi m \lambda f(\lambda)-2 \pi m f(\lambda)^{2} .
$$

This equation has the solution

$$
f(\lambda)=\lambda_{*} \frac{1+\chi \sqrt{\frac{\lambda_{*}}{\lambda}-1}}{\frac{\lambda_{*}}{\lambda}-2\left(1+\chi \sqrt{\frac{\lambda_{*}}{\lambda}-1}\right)},
$$

with an arbitrary constant $\chi$. Let us discuss the different solutions corresponding to different values of $\chi$ and their properties in turn.

- For $\chi \rightarrow \infty$, the function $f(\lambda)$ becomes constant:

$$
f(\lambda) \equiv-\frac{\lambda_{*}}{2}=\frac{1}{2 \pi m} \Longrightarrow \mu(t) \equiv \frac{\alpha}{2 m} \text {. }
$$

This solution corresponds to the pure bulk flow from the fixed point (I) to (II).

- For $\chi$ finite, let us expand the function $f(\lambda)$ for small (negative) $\lambda$,

$$
f(\lambda)=\lambda_{*}\left(\chi \sqrt{\lambda / \lambda_{*}}+\left(1+2 \chi^{2}\right)\left(\lambda / \lambda_{*}\right)+\cdots\right)
$$

We see that for all finite $\chi$, we have $f(0)=0$, so that the flow at $t=-\infty$ starts at $\lambda=\mu=0$.

- For $\chi=0, \mu$ grows at the same rate as $\lambda$ for $t \rightarrow-\infty$. This corresponds to the situation when $\mu$ itself is not turned on initially, but is just sourced by $\lambda$.

- For finite $\chi \neq 0, \mu$ grows as $\pi \alpha \lambda_{*} \chi \sqrt{C} \mathrm{e}^{\frac{2}{m} t}$ for $t \rightarrow-\infty$ which is the solution of the first-order approximation $\dot{\mu}=\frac{2}{m} \mu+\cdots$ to the boundary RG equation. $\chi$ thus determines how much and with which sign we turn on the boundary field in the beginning; indeed we have

$$
\chi=-\frac{1}{\pi \alpha \sqrt{\left|\lambda_{*}\right|}} \lim _{t \rightarrow-\infty} \frac{\mu(t)}{\sqrt{|\lambda(t)|}} .
$$

- For any finite $\chi$, the function $f(\lambda)$ has a pole at

$$
\lambda_{0}=\frac{\lambda_{*}}{2}\left(1-\frac{\chi}{\sqrt{1+\chi^{2}}}\right),
$$

at which the function $f(\lambda)$ diverges to $-\infty$ when $\lambda / \lambda_{*}$ approaches $\lambda_{0} / \lambda_{*}$ from below. Note that the perturbative fixed points (I) and (II) at $\mu=\alpha / 2 m$ correspond to $f=-\lambda_{*} / 2>0$, so the solution always runs away from the perturbative fixed point for large $t$.

- For finite $\chi<0$, the function $f$ first develops towards positive values, then reaches its most positive value

$$
f_{\max }=f\left(\lambda_{\max }\right)=-\frac{\lambda_{*}}{2}\left(1-\frac{1}{\sqrt{1+\chi^{2}}}\right) \quad \text { at } \quad \lambda_{\max }=\frac{\lambda_{*}}{2}\left(1-\frac{1}{\sqrt{1+\chi^{2}}}\right),
$$

and then runs towards $-\infty$. The maximal value $f_{\max }$ approaches $-\lambda_{*} / 2$ for $\chi \rightarrow-\infty$, so we can come arbitrarily close to the perturbative fixed point at $\mu=\alpha / 2 m$ by tuning $\chi$ to large negative values.

Similarly, the solution to the RG equation (4.24) is of the form

$$
\mu=\pi a_{2} f(\lambda(t)), \quad f(\lambda)=-\frac{m \lambda}{2}+\sqrt{-\lambda} \sqrt{1+m \pi \lambda} \chi,
$$

where $\chi$ is again an arbitrary real parameter. 


\section{References}

[1] Zamolodchikov A B 1987 Renormalization group and perturbation theory near fixed points in two-dimensional field theory Sov. J. Nucl. Phys. 461090

Zamolodchikov A B 1987 Yad. Fiz. 461819

[2] Zamolodchikov A B 1989 Integrable field theory from conformal field theory Adv. Stud. Pure Math. 19641

[3] Zamolodchikov A B 1986 Irreversibility of the flux of the renormalization group in a 2D field theory JETP Lett. 43730

Zamolodchikov A B 1986 Pis. Zh. Eksp. Teor. Fiz. 43565

[4] Affleck I and Ludwig A W W 1991 Universal noninteger 'ground state degeneracy' in critical quantum systems Phys. Rev. Lett. 67161

[5] Friedan D and Konechny A 2004 On the boundary entropy of one-dimensional quantum systems at low temperature Phys. Rev. Lett. 93030402 (arXiv:hep-th/0312197)

[6] Fredenhagen S, Gaberdiel M R and Keller C A 2007 Bulk induced boundary perturbations J. Phys. A: Math. Theor. 40 F17 (arXiv:hep-th/0609034)

[7] Fredenhagen S, Gaberdiel M R and Keller C A 2007 Symmetries of perturbed conformal field theories J. Phys. A: Math. Theor. 4013685 (arXiv:0707.2511 [hep-th])

[8] Gaberdiel M R, Konechny A and Schmidt-Colinet C 2009 Conformal perturbation theory beyond the leading order J. Phys. A: Math. Theor. 42105402 (arXiv:0811.3149 [hep-th])

[9] Gaberdiel M R and Lawrence A 2007 Bulk perturbations of $N=2$ branes J. High Energy Phys. JHEP05(2007)087 (arXiv:hep-th/0702036)

[10] Gaberdiel M R and Schlotterer O 2009 Bulk induced boundary perturbations for $N=1$ superconformal field theories J. Phys. A: Math. Theor. 42115209 (arXiv:0810.4719 [hep-th])

[11] Cherednik I V 1984 Factorizing particles on a half line and root systems Theor. Math. Phys. 61977 Cherednik I V 1984 Teor. Mat. Fiz. 6135

[12] Sklyanin E K 1988 Boundary conditions for integrable quantum systems J. Phys. A: Math. Gen. 212375

[13] Fring A and Köberle R 1994 Factorized scattering in the presence of reflecting boundaries Nucl. Phys. B 421159 (arXiv:hep-th/9304141)

[14] Ghoshal S and Zamolodchikov A B 1994 Boundary $S$ matrix and boundary state in two-dimensional integrable quantum field theory Int. J. Mod. Phys. A 93841 (arXiv:hep-th/9306002)

Ghoshal S and Zamolodchikov A B 1994 Int. J. Mod. Phys. A 94353 (Erratum)

[15] LeClair A, Mussardo G, Saleur H and Skorik S 1995 Boundary energy and boundary states in integrable quantum field theories Nucl. Phys. B 453581 (arXiv:hep-th/9503227)

[16] Lesage F, Saleur H and Simonetti P 1998 Boundary flows in minimal models Phys. Lett. B 42785 (arXiv:hep-th/9802061)

[17] Dorey P, Pocklington A, Tateo R and Watts G M T 1998 TBA and TCSA with boundaries and excited states Nucl. Phys. B 525641 (arXiv:hep-th/9712197)

[18] Dorey P, Runkel I, Tateo R and Watts G M T 2000 G-function flow in perturbed boundary conformal field theories Nucl. Phys. B $\mathbf{5 7 8} 85$ (arXiv:hep-th/9909216)

[19] Dorey P, Fioravanti D, Rim C and Tateo R 2004 Integrable quantum field theory with boundaries: the exact g-function Nucl. Phys. B 696445 (arXiv:hep-th/0404014)

[20] Dorey P, Pillin M, Tateo R and Watts G M T 2001 One-point functions in perturbed boundary conformal field theories Nucl. Phys. B 594625 (arXiv:hep-th/0007077)

[21] Bajnok Z, Palla L and Takacs G 2006 On the boundary form factor program Nucl. Phys. B 750179 (arXiv:hep-th/0603171)

[22] Castro-Alvaredo O A 2006 Boundary form factors of the sinh-Gordon model with Dirichlet boundary conditions at the self-dual point J. Phys. A: Math. Gen. 3911901 (arXiv:hep-th/0606269)

[23] Cardy J L 1989 Boundary conditions, fusion rules and the Verlinde formula Nucl. Phys. B 324581

[24] Recknagel A, Roggenkamp D and Schomerus V 2000 On relevant boundary perturbations of unitary minimal models Nucl. Phys. B 588552 (arXiv:hep-th/0003110)

[25] Pearce P A, Chim L and Ahn C 2001 Excited TBA equations: I. Massive tricritical Ising model Nucl. Phys. B 601539 (arXiv:hep-th/0012223)

[26] Pearce P A, Chim L and Ahn C 2003 Excited TBA equations: II. Massless flow from tricritical to critical Ising model Nucl. Phys. B 660579 (arXiv:hep-th/0302093)

[27] Ahn C and Rim C 1999 Boundary flows in the general coset theories J. Phys. A: Math. Gen. 32 2509 (arXiv:hep-th/9805101)

[28] Graham K 2002 On perturbations of unitary minimal models by boundary condition changing operators J. High Energy Phys. JHEP03(2002)028 (arXiv:hep-th/0111205) 
[29] Fredenhagen S and Schomerus V 2002 D-branes in coset models J. High Energy Phys. JHEP02(2002)005 (arXiv:hep-th/0111189)

[30] Chim L 1996 Boundary S-matrix for the tricritical Ising model Int. J. Mod. Phys. A 114491 (arXiv:hep-th/9510008)

[31] Ishibashi N 1989 The boundary and crosscap states in conformal field theories Mod. Phys. Lett. A 4251

[32] Cardy J L 1990 Conformal invariance and statistical mechanics Fields, Strings, Critical Phenomena (Proc. Les Houches Summer School 1988) ed E Brézin and J Zinn-Justin (Amsterdam: North-Holland) pp 169-246

[33] Runkel I 1999 Boundary structure constants for the A-series Virasoro minimal models Nucl. Phys. B 549563 (arXiv:hep-th/9811178)

[34] Fredenhagen S and Schomerus V 2003 On boundary RG-flows in coset conformal field theories Phys. Rev. D 67085001 (arXiv:hep-th/0205011)

[35] Fredenhagen S 2003 Organizing boundary RG flows Nucl. Phys. B 660436 (arXiv:hep-th/0301229)

[36] Petkova V B and Zuber J B 2001 Generalised twisted partition functions Phys. Lett. B 504157 (arXiv:hep-th/0011021)

[37] Fröhlich J, Fuchs J, Runkel I and Schweigert C 2007 Duality and defects in rational conformal field theory Nucl. Phys. B 763354 (arXiv:hep-th/0607247)

[38] Brunner I and Roggenkamp D 2008 Defects and bulk perturbations of boundary Landau-Ginzburg orbifolds $J$. High Energy Phys. JHEP04(2008)001 (arXiv:0712.0188 [hep-th])

[39] Graham K and Watts G M T 2004 Defect lines and boundary flows J. High Energy Phys. JHEP04(2004)019 (arXiv:hep-th/0306167)

[40] Bachas C and Gaberdiel M R 2004 Loop operators and the Kondo problem J. High Energy Phys. JHEP11(2004)065 (arXiv:hep-th/0411067)

[41] Oshikawa M and Affleck I 1997 Boundary conformal field theory approach to the critical two-dimensional Ising model with a defect line Nucl. Phys. B 495533 (arXiv:cond-mat/9612187)

[42] Kormos M, Runkel I and Watts G M T 2009 Defect flows in minimal models (arXiv:0907.1497 [hep-th])

[43] Affleck I and Ludwig A W W 1993 Exact conformal field theory results on the multichannel Kondo effect: single fermion Green's function, self-energy and resistivity Phys. Rev. B 487297

[44] Green D R, Mulligan M and Starr D 2008 Boundary entropy can increase under bulk RG flow Nucl. Phys. B 798491 (arXiv:0710.4348 [hep-th]) 